\title{
Biomedical application of photo-crosslinked gelatin hydrogels
}

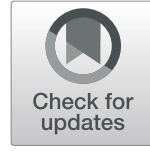

Lei Xiang and Wenguo Cui ${ }^{*}$

\begin{abstract}
During the past decades, photo-crosslinked gelatin hydrogel (methacrylated gelatin, GelMA) has gained a lot of attention due to its remarkable application in the biomedical field. It has been widely used in cell transplantation, cell culture and drug delivery, based on its crosslinking to form hydrogels with tunable mechanical properties and excellent bio-compatibility when exposed to light irradiation to mimic the micro-environment of native extracellular matrix (ECM). Because of its unique biofunctionality and mechanical tenability, it has also been widely applied in the repair and regeneration of bone, heart, cornea, epidermal tissue, cartilage, vascular, peripheral nerve, oral mucosa, and skeletal muscle et al. The purpose of this review is to summarize the recent application of GelMA in drug delivery and tissue engineering field. Moreover, this review article will briefly introduce both the development of GelMA and the characterization of GelMA. Finally, we discuss the challenges and future development prospects of GelMA as a tissue engineering material and drug or gene delivery carrier, hoping to contribute to accelerating the development of GelMA in the biomedical field.
\end{abstract}

Keywords: Gelatin, Gelatin methacryloyl, Biomedical application, Hydrogels, Photo crosslink

\section{Introduction}

Gelatin attracts more and more attention in the biomedical field, owing to its unique characteristics such as biocompatibility, solubility, low immunogenicity, low cytotoxicity, easy acquirement, and so on $[1,2]$. As a kind of natural hydrogel, gelatin possesses the unique structure, similar to native extracellular matrix (ECM), which is very suitable for in vitro cell culture [3], and it also can become even several times larger than its original volume and weight without dissolution in the water. Gelatin contains the arginine-glycine-aspartic acid (RGD) peptide sequence and the matrix metalloproteinase (MMP) degradable motifs, the one which is beneficial for cellular growth, and the other controls cell enzymatic degradation $[4,5]$.

To further improve this materials, Van den Bulcke and coworkers firstly modified gelatin hydrogels with

\footnotetext{
* Correspondence: wgcui80@hotmail.com

Shanghai Institute of Traumatology and Orthopaedics, Shanghai Key Laboratory for Prevention and Treatment of Bone and Joint Diseases, Ruijin Hospital, Shanghai Jiao Tong University School of Medicine, 197 Ruijin 2nd Road, Shanghai 200025, P. R. China
}

\section{Springer Open}

(c) The Author(s). 2021 Open Access This article is licensed under a Creative Commons Attribution 4.0 International License, which permits use, sharing, adaptation, distribution and reproduction in any medium or format, as long as you give appropriate credit to the original author(s) and the source, provide a link to the Creative Commons licence, and indicate if changes were made. The images or other third party material in this article are included in the article's Creative Commons licence, unless indicated otherwise in a credit line to the material. If material is not included in the article's Creative Commons licence and your intended use is not permitted by statutory regulation or exceeds the permitted use, you will need to obtain permission directly from the copyright holder. To view a copy of this licence, visit http://creativecommons.org/licenses/by/4.0/. tural and related mechanical properties. A few amino acid residues (about $5 \%$ of molar ratio) were involved during the process of synthesizing GelMA [6] and the RGD motifs will not react with MA [7-9], which means the functions of RGD and MMP degradable motifs are still reserved. GelMA used in the biomedical field is mainly derived from animal skin and can be divided into two types: type A GelMA derived from porcine skin and type B GelMA derived from bovine skin.

GelMA not only maintains good bio-compatibility, low immunogenicity, and the ability to promote cellular growth, but also obtains the property of forming 3D structure under the radiation of UV with the presence of a photoinitiator (because the side groups of methacrylamide and methacrylate of gelatin chain were polymerized to obtain cross-linked gelatin network). Compared with other kinds of hydrogels, the most prominent advantage of GelMA is that the mechanical properties can be adjusted by multiple factors, such as GelMA concentration, the kinds of photoinitiator (Irgacure 2959, lithium phenyl-2,4,6-trimethylbenzoylphosphinate and VA- 
086 are typical photoinitiators used for UV crosslinking), photocrosslinking times, ultraviolet (UV) dose during photoinitiation and so on [10-12], according to actual needs. Crosslinking which is induced by light in a photo-initiated polymerization reaction, is a typical method used to alter physicochemical properties of GelMA. When exposed to the UV light in the presence of a photoinitiator, the methacrylamide and methacrylate side groups on the GelMA chains will polymerize via radical addition-type polymerization to form a $3 \mathrm{D}$ network structure.

Based on its 3D structure and the RGD and MMP degradable motifs, GelMA has been widely applied in cell culture field, which represents the method of cell culture shift from monolayers (2D) to the third dimension and also provides prerequisites for the application of GelMA in tissue engineering. In the tissue engineering field, autografts and allografts have been widely utilized for accelerating healing and functional recovery of damaged areas [13]. However the resource of donor organs can not satisfy the clinic demand $[14,15]$. As a kind of promising material, GelMA hydrogels combines the advantages of natural and synthetic hydrogels, becoming a research focus in the tissue engineering field. Complex living tissue culture, repair and regeneration remain a big challenge. Take bone defect repair as an example, while the graft fills the defect area, whether the blood supply of the defect area, peripheral nerves, sensory neurons and muscles can regenerate and heal as soon as possible affects the final prognosis. GelMA not only can repair defect area without severe inflammatory response (owing to its unique bio-compatibility and mechanical tenability), but also can promote the peripheral neurons and vascular regeneration (owing to RGD, MMP degradable motifs and ECM-like micro-environment).

In this review, we focus on recent studies related to the biomedical application of GelMA hydrogel including its basic characterization and drug delivery, as well as tissue engineering field. Finally, we will discuss the challenges and future development prospects of GelMA as a tissue engineering material and drug delivery carrier (Scheme 1).

\section{Basic synthetic procedures and influencing factors on GelMA hydrogels characterization}

\subsection{Basic synthetic steps}

Since GelMA was first synthesized in 2000, many researchers have optimized and adjusted the synthesis steps according to their own needs, but their basic synthesis steps are similar. Next, we will briefly introduce the synthesis method of GelMA (Fig. 1). In general, the GelMA synthesis is that gelatin is modified with methacrylic anhydride (MA) to graft unsaturated bounds (methacrylamide groups and methacrylate groups) onto the molecular chains $[7,17,18]$, and the hydrogels are fabricated by cross-linking of solubilized GelMA with the presence of a photoinitiator.

Step 1: Dissolve the gelatin in the phosphate buffer $(\mathrm{pH}=7.5)$ at $50^{\circ} \mathrm{C}$, then add the MA and constantly stir for $1 \mathrm{~h}$ to ensure sufficient reaction of MA and gelatin (The reactivity of amine and hydroxyl groups and the amount of MA influence the degree of substitution [19]).

Step 2: The reaction product is dialyzed against deionized water for more than $24 \mathrm{~h}$ at $40^{\circ} \mathrm{C}$, to remove possible residual toxic impurity (including residue of methacrylic anhydride and the byproducts of reaction, etc.).

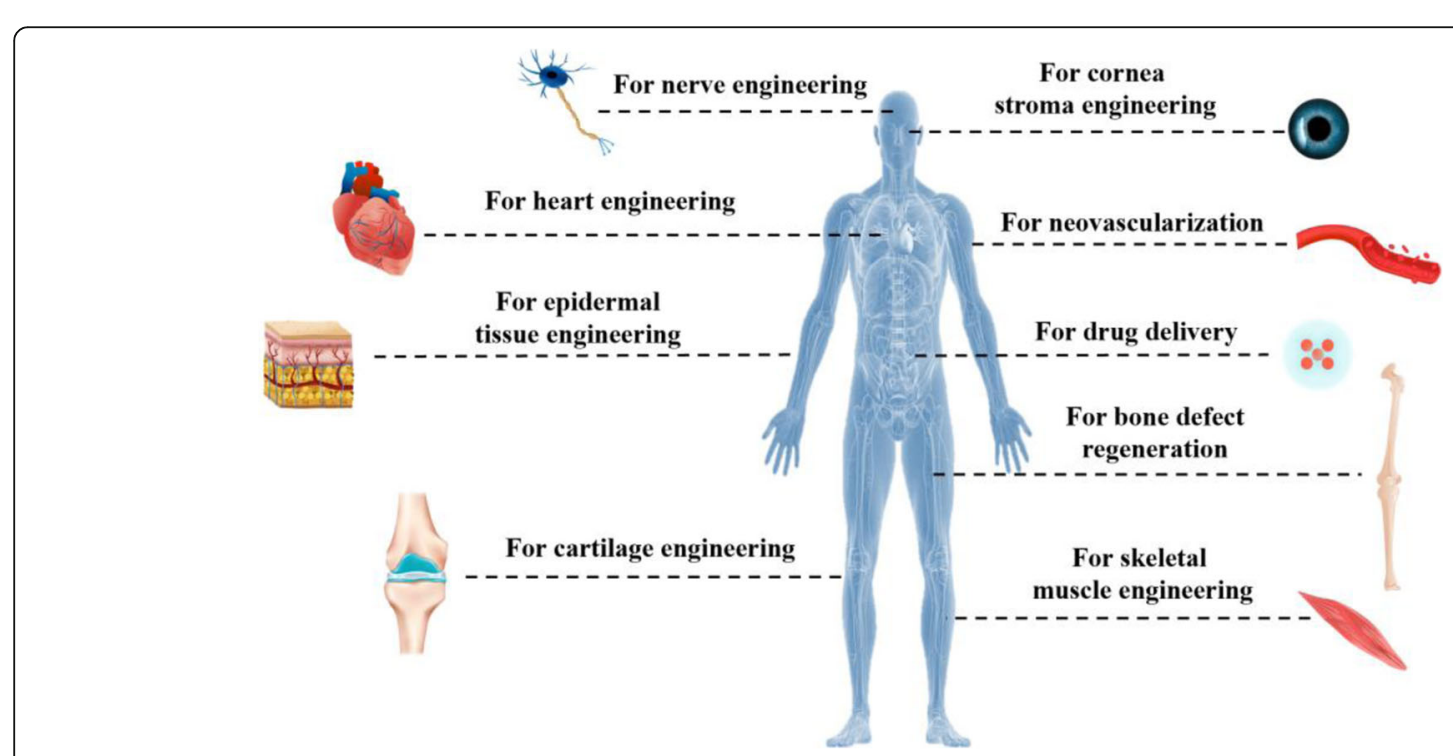

Scheme 1 Photo-crosslinked gelatin hydrogel (methacrylated gelatin, GeIMA) has been widely applied in various biomedical fields, such as the repair and regeneration of bone, heart, cornea, epidermal tissue, cartilage, vascular, nerve, skeletal muscle and drug delivery and so on 

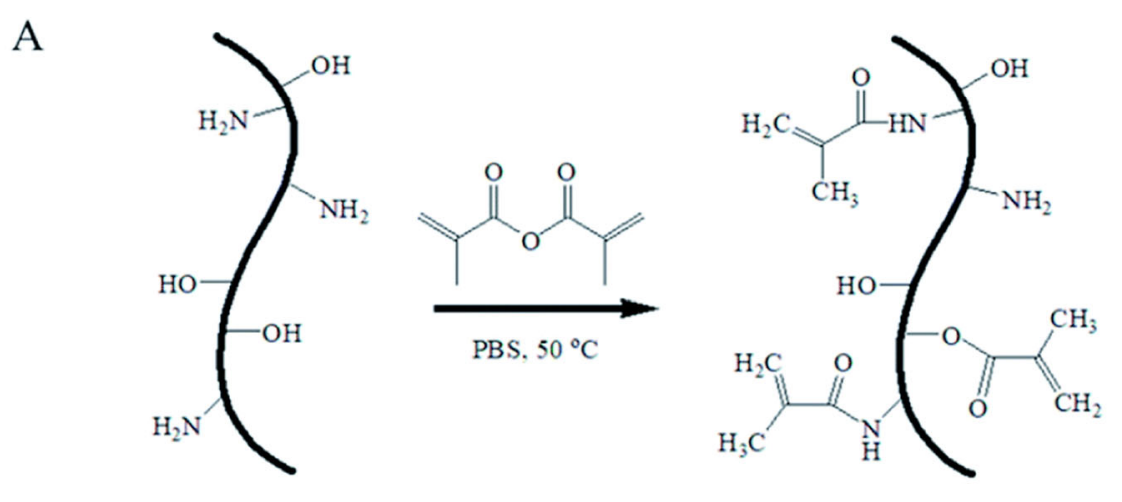

B

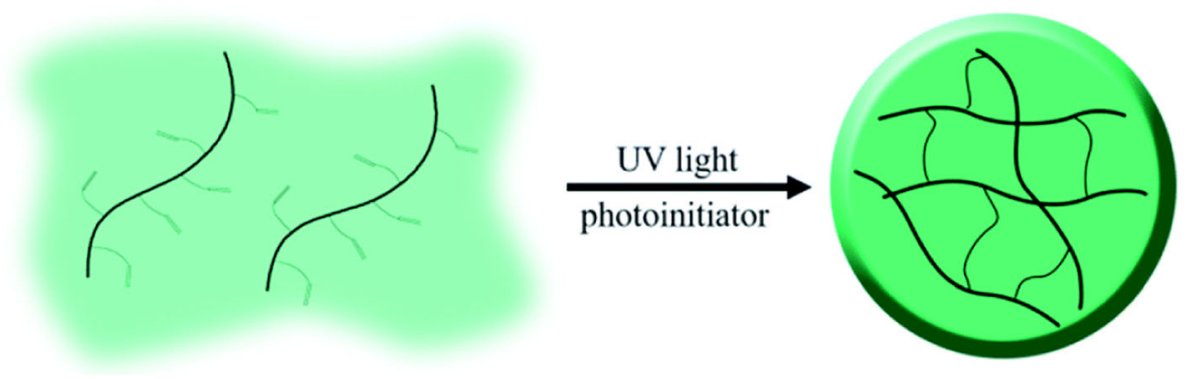

Fig. 1 Diagram of GeIMA synthesis and photocrosslinking. Reproduced with permission [16]. Copyright (2019), The Royal Society of Chemistry

Step 3: Freeze drying and storing the product in the refrigerator until use.

Step 4: Dissolve the GelMA in the deionized water at $40{ }^{\circ} \mathrm{C}$, add moderate photoinitiator and $\mathrm{NaN}_{3}$ as antimicrobial agents.

Step 5: Pour the mixture into a pre-prepared container and cool to room temperature,and exposure to radiation of UV for a given.

Step 6: After storing the cast at $4{ }^{\circ} \mathrm{C}$ for a given period, remove the pre-prepared container, and GelMA hydrogels are obtained.

\subsection{Influencing factors on GelMA hydrogels characterization \\ 2.2.1 Mechanical properties}

As the material used for repair and regeneration of various organs and tissue, the tunable mechanical properties of GelMA is vitally important. In previous studies, we have reported the relationship between the concentration of GelMA prepolymer solution and mechanical properties [20]. Through increasing the concentration of GelMA prepolymer solution, both Young's modulus and compressive modulus shift from a few $\mathrm{kPa}$ to a few hundred $\mathrm{kPa}$. Chen et al. [21] explain the the effects of the degree of methacryloyl substitution (DS) on GelMA mechanical properties. Increasing the the degree of methacrylation substitution could enhance the compression modulus of GelMA hydrogel. The sources of the gelatin used for GelMA fabrication also have influence on its mechanical properties. Type B (from bovine skin) GelMA has a slightly higher DS compared to type A (from porcine skin) GelMA, especially in a lower feed ratio of methacrylic anhydride to gelatin, which leads to better mechanical properties of type B GelMA (under the same synthesis conditions) [17]. The UV exposure time and elastic modulus are positively correlated, which is reported by Schuurman et al. [22]. Colosi et al. [23]. have reported that increase the intensity of UV light or prolong the exposure time would strengthen the hydrogel's mechanical properties.

\subsubsection{Porosity}

Appropriate porosity can provide enough space for cell growth, ensure that cells can get sufficient nutrition and remove metabolic waste in time [24] so that porosity plays a crucial role in cell culture. It has been reported that the degree of substitution of the amino group [21, $25]$, the concentration of GelMA $[10,24]$ and the and the cooling rate [26] will affect the porosity of GelMA. Overall, the above three factors are negatively correlated with the porosity and the average size of the pore.

\subsubsection{Swelling property}

The swelling property of a hydrogel, which is influenced by hydrogel pore size, is helpful for the diffusion of healing factors and highly affects the mechanical properties 
of scaffolds [27-29]. It can be evaluated by weighing method. We and other researchers have reported that the increasing GelMA concentrations from 5\% to 20\% will lead to the decrease of swelling ratios from 1500\% to $500 \%[10,20]$. The swelling property can also be modified by adding organic or inorganic ingredients. Kong et al. found that the add grid fibers into GelMA would result in the swelling ratio of GelMA hydrogel decrease [28]. Jihwan and Bumsang [30] studied the $\mathrm{pH}$-responsive behavior of GelMA and concluded that the swelling rate of GelMA under low $\mathrm{pH}(<5)$ environment was significantly lower than that under high $\mathrm{pH}$ conditions $(>5)$.

\subsubsection{Degradation}

Uncontrolled rapid degradation is a major challenge for GelMA in the field of biomedicine [31], for which many researchers are committed to finding ways to control the degradation of GelMA. Our previous study [20] have reported the influence of GelMA concentration on degradation. The degradation rate increased with the decrease of GelMA concentration. Complete degradation time of $20 \%$ GelMA was more than 8 weeks, while that of $5 \%$ GelMA was less than 3 days. Srikumar [32] found that the degree of methacryloyl substitution (DS) significantly affects degradation. Michael addition reaction is another method to control the degradation of hydrogels [33, 34], which is based on nucleophilic addition between $\mathrm{Mi}$ chael acceptors and donors. Nicolas et al. produced a film by generating disulfide bindings, which resulted in high resistance against enzymatic degradation [35].

\section{GelMA for drug delivery}

How to improve the utilization rate of drugs in the body has been an eternal topic in the field of biomedicine. Besides structural transformation and optimization of the drug itself, further optimizing the drug delivery system to achieve the goal of controlled release of the drug is also a feasible way. Due to its unique 3D structure and electrostatic interactions, GelMA has been widely used in the drug delivery field. Based on these properties, many researchers have combined the GelMA with other materials, such as nanomaterials, liposome, and made great progress in the field of drug delivery and controlled drug release.

Ning et al. [36] reported that GelMA can achieve sustained release of abaloparatide for up to 10 days. Julia et al. [37] reported the application of GelMA in BMP-2 delivery and promoted differentiation of human adiposederived stem cells. By comparing the loading of different concentrations of BMP-2, they found that the dose of growth factor in this system did not have much effect on efficiency (BMP-2 loaded at concentrations of $25 \mu \mathrm{g} / \mathrm{ml}$ result in $27.0 \%$ loaded growth factor maintained in the hydrogels for at least seven weeks). Margaux et al. [38] developed a novel paclitaxel-based Abraxane delivery system based on GelMA. This drug delivery system can achieve high encapsulation efficiency (96\%) without affecting its mechanical properties and the rate of drug release can be adjusted by controlling the concentration of GelMA (lower GelMA concentration will lead to longer drug release time).

To meet different delivery conditions of different drugs, researchers also combine GelMA with other materials. Cheng et al. [39] combined liposome with hydrogel and fabricated GelMA-lip scaffold, which improved the mechanical properties and realized the drug controlled release in stages (the early release of hydrophilic drugs, the mid-term release of bio-active macromolecule and long-term release of liposoluble medicine). In the rat femoral defect model, this new scaffold can effectively promote the formation of new bone and new blood vessels. Nanomaterials have also been used to improve the performance of GelMA in drug delivery. Samanipour et al. [40] fabricated nanocage-laden GelMA hydrogels to overcome the shortcoming of GelMA in uncontrollable drug release. The drug release of nanocage-laden GelMA hydrogels can be controlled by adjusting $\mathrm{pH}$ value (this hydrogels shows drug storage potential at $\mathrm{pH}$ 7.4 and presented the ability of controlled-release at $\mathrm{pH}$ 2). Kang et al. [41] have also developed $\mathrm{pH}$-sensitive nanogels derived from fish gelatin, which realized controllable drug release at lower $\mathrm{pH}$. Khashayar et al. [42] developed GelMA/chitosan nanoparticles composite hydrogel and achieved the goal of promoting cell proliferation and sustained release of bFGF. Various polymers are also used to enhance the mechanical properties of GelMA to better meet the needs of controlled drug delivery. Salise et al. [43] incorporate poly (3,4-ethylenedioxythiophene) / poly (styrenesulfonate) (PEDOT/PSS) with GelMA and successfully fabricated electro-responsive hydrogels, which can promote drug release by applying voltage. $\mathrm{Li}$ et al. [44] fabricated core-shell microparticles by using GelMA aqueous solution and PLGA oil solution, which can co-delivery both of DOX and CPT for cancer treatment. This structure can ensure that the drug-loaded in the GelMA core can not be released until the PLGA shell is degraded and the size of the core and the thickness of the shell can be adjusted according to actual needs through microfluidic technology.

For some genetic diseases and cancers, modifying cells to change their gene expression is a very effective treatment. Paresh et al. [45] provided an efficient method for in situ gene delivery via PEG diacrylate matrices. They have combined PEGDA based cryogels with GelMA and PLL to improve cell adhesion and retain viruses for localized gene delivery and found that this novel method 
showed higher transduction efficiency and improved retention of green fluorescent protein (GFP) lentiviruses. Some organic ingredients are also used to modify GelMA. Lai et al. developed GelMA/CBMA (carboxybetaine methacrylate) which can effectively control the release of biological macromolecules $(>250 \mathrm{KDa})$ [46]. Sun et al. [47] combined the beneficial properties of GelMA and arginine through photocrosslinking technology and increased protein loading level and achieved the sustained release of BMP-2 protein (BMP-2 release from GelMA/Arg was completed around 5-10 days compared with pure GelMA 2-3 days).

Because magnetic nanoparticles (MNPs) can perform directional motion in a magnetic field, many researchers use it as an engine for the directional transport of drugs and developed smart drug carriers. Jalili et al. [48] fabricated poly NIPAM-co-AM/MNPs/GelMA for localized, on-demand delivery of doxorubicin (DOX). People can control the release of drugs by adjusting the temperature and magnetic field. Mei et al. [49] fabricated microswimmer, based on GelMA, and integrate magnetoelectric nanoparticles (MENPs) into the microswimmers serve as the engine. In a specific magnetic field, it can achieve targeted delivery of cells to neurons and the MENPs can convert magnetic signals into electrical signals, which can induce the differentiation of neuronal cells. Microneedles (MNs) is a kind of novel technology platform for sustained drug release, Luo et al. [50] used GelMA as raw materials to fabricate an innovative biodegradable MNs. This MNs can effectively penetrate the stratum corneum of the skin and the drug release rate can be controlled by adjusting the time of photocrosslinking (longer crosslinking time leads to decreased release rates).

Besides all the above, GelMA also has interesting applications in other fields of biomedicine, such as testing drug toxicity and filtrating drugs. Lu et al. [6] developed the decellularized liver matrix (DLM)-GelMA based biomimetic liver tumor-on-a chip model via microfluidic technology. This novel model can better mimic the native tumor micro-environment and obtain better capability to promote cell viability and proliferation compared with pure GelMA, which provided new ideas for the study of pathology and pharmacology.

\section{GelMA for neovascularization}

For cells and tissues with material exchange and metabolic functions, the mature vascular network can ensure adequate supply of nutrients and timely removal of metabolic waste, thereby ensuring the normal operation of tissues and organs and avoiding tissue ischemic necrosis. The preparation of a fully functional 3D vascular network is a major challenge in the field of tissue engineering and a prerequisite for the further development of many tissue engineering therapies. Because of its unique 3D structure and good bio-compatibility, GelMA is widely used as a culture carrier for HUVECs and other cells, in order to achieve further breakthroughs in neovascularization and these applications are summarized in the Table 1.

GelMA hydrogel has been proven to be a cell carrier and promote long-term cell survival. Based on this, Choi

Table 1 The recent application of GelMA in neovascularization

\begin{tabular}{|c|c|c|c|}
\hline $\begin{array}{l}\text { Polymer } \\
\text { scaffold }\end{array}$ & Cell & Achievement & Reference \\
\hline GelMA/ALG & HUVECS & $\begin{array}{l}\text { Subsequent transplantation of the micro-hydrogel into a hindlimb ischemia model } \\
\text { effectively attenuated the ischemia condition. }\end{array}$ & [51] \\
\hline GelMA & SCAP HUVECS & $\begin{array}{l}\text { SCAP cells spread in close contact with endothelial networks and expressed alpha } \\
\text { smooth muscle actin aSMA expression was also apparently higher }\end{array}$ & [52] \\
\hline PCL/GelMA & HUVECS & $\begin{array}{l}\text { Exhibit desirable scaffold characteristics as 3D cell microenvironments for } \\
\text { supporting endothelial cell attachment and growth }\end{array}$ & [53] \\
\hline GelMA/ALG & HUVECS & $\begin{array}{l}\text { The endothelial cells encapsulated inside the bioprinted microfibers can form the } \\
\text { lumen-like structures resembling the vasculature over the course of culture for } 16 \text { days }\end{array}$ & [54] \\
\hline PEG-GelMA & HUVECS & $\begin{array}{l}\text { Exhibits enhanced cell attachment similar to that of native vascular endothelial cells } \\
\text { and demonstrated rapid and directionally persistent migration. }\end{array}$ & {$[55]$} \\
\hline GelMA & HUVECS & $\begin{array}{l}\text { Encapsulated HUVECs cultured in fully supplemented medium attached and proliferated } \\
\text { within the GeIMA fiber and demonstrated spontaneous cord-formation }\end{array}$ & {$[56]$} \\
\hline GelMA & ECFCs MSCs & $\begin{array}{l}\text { Show a rapid formation of functional anastomoses between the bioengineered human } \\
\text { vascular network and the mouse vasculature. }\end{array}$ & [21] \\
\hline GelMA & ECFCs MSCs & $\begin{array}{l}\text { The implanted human cells generated an extensive vascular network that formed functional } \\
\text { anastomoses with the host vasculature and was uniformly distributed throughout the construct. }\end{array}$ & [27] \\
\hline GelMA/C & HUVECS HCASMCS & Controllable mechanical strength, high cell/tissue adhesion, and excellent bio-functionalization. & [57] \\
\hline GelMA & $\begin{array}{l}\text { Murine } 10 T 1 / 2 \text { cells } \\
\text { HUVECs }\end{array}$ & A rapid approach to engineer a user-defined multicellular vascular chip & [58] \\
\hline
\end{tabular}


et al. [51] prepared GelMA / ALG hydrogel via electrospraying method and seeded HUVECs cells on it which can express hVEGF to promote angiogenesis. Their in vitro cell analysis indicate that $3.5 \mathrm{w} / \mathrm{v}$ \% GelMA hydrogel is optimal concentration resulted to enhance angiogenic response of endothelial cells. This novel GelMAbased platform can effectively improve the ischemic condition of the mouse hindlimb ischemia model. Ensuring that the endothelial cells are homogenously located on the 3D structure and rapidly form a confluent endothelial monolayer to quickly build functional endothelialized blood vessels is a major challenge in treating dangerous vascular diseases with vascular graft therapy. Zhao et al. [53] combined a shape-memory polymer and electrospun nano-membrane to design a new type of scaffold that can spontaneously curl from a 2D plane, where HUVECs can be seeded, into a 3D structure at normal body temperature $\left(37^{\circ} \mathrm{C}\right)$. This kind of scaffold realizes the programmed deformation from $2 \mathrm{D}$ to $3 \mathrm{D}$ while ensuring the homogenously distribution of endothelial cells, and its related properties can be adjusted by changing the nanomembrane.

\section{GelMA for nerve engineering}

Nerve, as the main conduction pathway of electrical and chemical signals in the body, is the main way for the brain to coordinate the functions of various organs and receive sensory signals. Severe trauma or endocrine disease can cause nerve fiber lesions. After the nerve is damaged, the body will automatically mobilize the fiber cells to repair itself, but this also causes the repaired nerve to lose its original function and cause local or systemic sensory or motor disorders [66]. At present, the main way to deal with serious nerve injury (defect is more than $5 \mathrm{~mm}$ ) clinically is to use autologous nerve transplantation [67], but this method also has many defects, such as damage to the original nerve function and the source of the graft can not satisfy the clinical needs. In the following parts we will introduce the recent application of GelMA in the nerve regeneration field and they are summarized in Table 2.

In order to prepare nerve repair materials with better performance, various technologies, such as $3 \mathrm{D}$ printing technology and Electrospinning technology are used to further optimize the GelMA hydrogels. Tao et al. [59] used 3D printing technology to further improve the efficiency of conduits in repairing peripheral nerve and fabricate GelMA/MPEG-PCL loaded with XMU-MP-1, which is a Hippo pathway inhibitor. They further explored the potential influence of XMU-MP-1 in nerve regeneration. From their experimental results we can conclude that XMU-MP-1 treatment promoted both the migration of Schwann cells and the mRNA expression of various neurotrophic factors with the XMU-MP-1 concentration increased. Cui et al. [62] used Electrospinning technology to construct a novel GelMA hydrogel electrospun fibers for spinal cord regeneration. This novel material has a good swelling rate and lower Young's modulus and can minic the components and microstructures of spinal cord better. The result of quantitative measurement of light density proved the ability of GelMA hydrogel electrospun fibers in promoting the migration of neural stem cells and induces their differentiation into neuronal cells. From the result of immunofluorescence staining, we can easily found the amount of Tuj-1-labeled neurons is significantly larger in GelMA group when compared with gelatin group and control group.

In view of the fact that the mechanical properties of GelMA can be adjusted from several Pa to thousands $\mathrm{Pa}$, what is the optimal stiffness of GelMA that can simulates the natural nerve regeneration environment better

Table 2 The recent application of GelMA in nerve regeneration engineering

\begin{tabular}{|c|c|c|c|}
\hline Polymer scaffold & Cell & Achievement & Reference \\
\hline GelMA/MPEG-PCL & Schwann Cells (S16) and HUVECs & $\begin{array}{l}\text { Promote Schwann cells proliferation and migration by inhibiting } \\
\text { Hippo signaling pathways to accelerate repair of nerve defects. }\end{array}$ & {$[59]$} \\
\hline GelMA-DA & NSCs & $\begin{array}{l}\text { Exhibits hierarchical structures for supporting stem cell growth and } \\
\text { improves neural differentiation of NSCs. }\end{array}$ & {$[60]$} \\
\hline GelMA & iNSCs & $\begin{array}{l}\text { Significantly promote functional recovery and the inflammation was } \\
\text { decreased by reducing activated macrophages/microglia. }\end{array}$ & {$[61]$} \\
\hline GelMA & BMSCs & $\begin{array}{l}\text { It has a swelling rate and compressive modulus which are more suitable } \\
\text { for nerve growth. It also can effectively reduce the formation of scar tissue } \\
\text { at the injury site while promoting vascular regeneration and directional } \\
\text { differentiation of neural stem cells. }\end{array}$ & {$[62]$} \\
\hline GelMA & $\mathrm{PC} 12$ & The $10 \%$ GelMA hydrogel is optimal for nerve regeneration of PC12 cell & {$[63]$} \\
\hline GelMA/EHS & / & $\begin{array}{l}\text { Shows a consistent tendency of improvement with time } \\
\text { and speed up the repair of nerve defects. }\end{array}$ & {$[64]$} \\
\hline GelMA/MeTro & Schwann Cells & $\begin{array}{l}\text { Compared with the control group, it has better cell adhesion ability, which } \\
\text { can promote cell proliferation and differentiation while inhibiting the } \\
\text { occurrence of inflammatory response. }\end{array}$ & {$[65]$} \\
\hline
\end{tabular}


and accelerates nerve outgrowth is a focus issue. In order to find out the answer to this question, Wu et al. [63] did an in-depth study. They selected the PC12 cells as the representative neurone and adjusting the concentration of GelMA to fabricate Hydrogel with the variation of stiffnesses. They found that the cell spreading area and the neuritis length first increased and then decreased with the stiffness increased and the cell adhesion rate is negatively correlated with stiffness. They came to the final conclusion that the 10\% GelMA hydrogel is optimal for nerve regeneration of $\mathrm{PC} 12$ cell.

\section{GelMA for bone defect regeneration}

In recent years, traffic accidents and serious trauma events have occurred frequently, resulting in more and more serious cases of bone tissue defects in the clinic. Bone tissue defects repair is a long process, which is mainly divided into three stages: Fristly, organization of hematoma stage, the body will clear the hematoma and produce a large number of fibroblasts. Secondly, the period of callus formation, new bone forms around the lesion area and forms callus and the fracture has reached the stage of clinical healing. Thirdly, bone marrow cavity recanalizes during callus shaping. This series of processes requires the simultaneous coordination of blood supply and neurotrophic regeneration. At present, the treatment of such cases in the clinic is mainly to fill the defect area through the implantation of autograft or allograft to ensure its restoration of morphological integrity and inject growth factors to promote the formation of new bone and accelerate the healing of fractures. However, the current source of autograft or allograft can not satisfy the huge clinical needs, and grafts tend to cause inflammation response and lead to complications. Benefiting from the adjustable mechanical properties of GelMA (which can meet the mechanical requirements of load-bearing tissues) and good bio-compatibility (reducing the inflammatory response in the damaged area), it has been paid significant attention in the field of bone tissue repair and more and more researchers have also begun to explore its application in bone tissue repair. Various types of scaffolds, such as GelMA/PEGDA [68, 77], GelMA/MBG [69, 75, 76], GeLMA/liposome [70, 86] et al. have been developed for bone defect regeneration and the recent applications of GelMA in the field of bone tissue are summarized in Table 3 .

3D printing technology has received much attention in recent years. Liu et al. [71] have applied 3D printing technology to the preparation of biological scaffolds and have successfully developed a kind of multi-layered GelMA/nHA scaffold for bone defect repair (Fig. 2). The results of animal experiments can prove that this material can restore the integrity of the damaged bone surface and can deposit extracellular matrix and abundant collagen type II.

CT is often used to diagnose and reexamine skeletal lesions, but the CT signal becomes weak if the main composition of materials is water. In order to solve the imaging problem of the hydrogel, Celikkin et al. [89] fabricated 3D printed GelMA and GelMA-AuNPs scaffolds with enhanced visibility for $\mu \mathrm{CT}$ imaging (Fig. 3).

The repair of large bone defects is not only to fill the defect area with grafts but also to induce the regeneration of peripheral vessels and nerves to ensure adequate nutrition during the regeneration of bone tissue. Periosteum not only plays an important role in maintaining the morphological integrity of bones, but also plays a very important role in promoting mineralization and promoting the formation of new bones and new blood vessels. In order to promote blood vessel regeneration and strengthen osteogenesis in bone reconstruction, Cui et al. have developed a novelty inorganic strengthened gelatin hydrogel membrane GelMA-G-MBGNs [75]. After testing, this new artificial periosteum significantly enhances the mechanical properties and structural stability of the material. And in animal experiments, this material also has an excellent performance in promoting bone tissue and vascular regeneration (Fig. 4), which plays a vital role in promoting the repair of large bone injuries.

Bone mineralization refers to the biochemical process in which inorganic minerals are deposited in the organic matrix of bone. In this process, calcium and phosphorus form apatite which is chelated with organic matter to form bone. Mineralization is an important part of bone tissue repair. Bioactive glass (BG), as the third-generation biomaterial, has good bio-compatibility, bone conductivity and the ability to induce mineralization. $\mathrm{BG}$ can release $\mathrm{Si}, \mathrm{Ca}$, and $\mathrm{P}$ ions in the environment of biological fluids to stimulate signaling pathways related to osteogenesis and blood vessel production, and promote bone mineralization and regeneration. Zheng et al. [72] reported their research on fabricating GelMA/BG scaffold via sequential gelation and UV approach. This scaffold shows better apatite-forming ability (Fig. 5a) and better ability of cell proliferation (Fig. 5b) with the concentration of BG increased. Kwon et al. are also devoted to the research of BG/GelMA [88], they report that the mechanical properties and the ability to induce bone differentiation of this material will increase with increasing concentration of BG. In addition, in vivo testing according to the mouse skull defect model shows that the highest concentration $(2.5 \mathrm{w} / \mathrm{w} \%)$ of bioglass nanoparticles incorporated into the GelMA cryogel induced the most bone formation.

In recent years, stem cell transplantation technology has been increasingly used for the treatment of various 
Table 3 The recent applicati on of GelMA in bone tissue regeneration

\begin{tabular}{|c|c|c|c|}
\hline Polymer scaffold & Cell & Achievement & Reference \\
\hline GelMA/PEGDA & $\begin{array}{l}\text { MC3T3-E1 } \\
\text { L929 }\end{array}$ & $\begin{array}{l}\text { Improves mechanical properties and structural stability and promote the proliferation } \\
\text { and adhesion of osteoblast cell. }\end{array}$ & [68] \\
\hline 26SCS/MBG/GelMA & $\begin{array}{l}\text { hBMSCS } \\
\text { HUVECS }\end{array}$ & Enhances the GFs' bio-activity and decreases antagonism effects of the noggin. & [69] \\
\hline DFO/liposome/GelMA & / & Achieve the goal of controlling the release of DFO. & [70] \\
\hline GelMA/nHA & BMSCs & $\begin{array}{l}\text { The regenerated tissue in the defect area can restore its morphological integrity } \\
\text { and ensure the smoothness of the tissue surface. }\end{array}$ & [71] \\
\hline BG/GelMA & mBMSCs & $\begin{array}{l}\text { significantly improve the bio-activity and stability, promote cell attachment, } \\
\text { proliferation and osteogenic differentiation. }\end{array}$ & [72] \\
\hline GelMA/B-TCP & hASCs & $\begin{array}{l}\text { Based on achieving stronger bone tissue repair ability, it also strengthens the } \\
\text { mechanical strength and bio-compatibility. }\end{array}$ & [73] \\
\hline nAg/HNTs/GelMA & hPDLSCs & $\begin{array}{l}\text { While ensuring that it can promote the differentiation of hPDLSCs under } \\
\text { inflammatory conditions, it also has excellent antibacterial capabilities. }\end{array}$ & [74] \\
\hline GeIMA-G-MBGN & MC3T3-E1 & $\begin{array}{l}\text { Improve mechanical properties and structural stability and have a long-term ion } \\
\text { release function, which makes it more suitable for cell adhesion and proliferation. }\end{array}$ & [75] \\
\hline GelMA/MBGNs-rhBMP-2 & BMSCs & $\begin{array}{l}\text { It can maintain the level of rhBMP-2 in vivo for a long time, which can ensure } \\
\text { BMSCs proliferation and differentiation. }\end{array}$ & [76] \\
\hline GelMA/PEGDA & PDLSCS & Shows the good ability to promote cell reproduction and inducing cell differentiation. & [77] \\
\hline HNTs/GelMA & hDPSCs & $\begin{array}{l}\text { Under the premise of ensuring that cell behavior is not affected, the mechanical } \\
\text { properties are further strengthened. }\end{array}$ & [78] \\
\hline GelMA/OGP & MC3T3-E1 & $\begin{array}{l}\text { Accelerate the healing of bone injury areas and the formation of new bone from } \\
\text { the genetic level (improve the expression of related genes,such as BMP-2). }\end{array}$ & [79] \\
\hline PACG/GelMA & hBMSCs & $\begin{array}{l}\text { Show outstanding compressive strength, large Young's modulus, and high } \\
\text { compression modulus and facilitate concurrent regeneration of cartilage. }\end{array}$ & [80] \\
\hline GelMA/ALN & hFOBCs & $\begin{array}{l}\text { It can effectively increase the expression of related genes and promote } \\
\text { bone mineralization and hFOBCs differentiation. }\end{array}$ & [81] \\
\hline DN/GelMA & MSCs & $\begin{array}{l}\text { Enhance stiffness and toughness and preferentially guide MSCs toward } \\
\text { directed differentiation in vitro. }\end{array}$ & [82] \\
\hline GeIMA/HANW & BMSCs & $\begin{array}{l}\text { Improved the compressive strengths and mechanical stability of the } \\
\text { composite cryogels and allow cell migration within the matrix }\end{array}$ & [83] \\
\hline OCP/GelMA & HUVECS & Promote both osteoblastic and angiogenic differentiation. & [84] \\
\hline nHAp/GelMA & Preosteoblast cells & $\begin{array}{l}\text { Significantly increase the structural stability and mechanical properties } \\
\text { and provide optimal conditions for cell adhesion and differentiation. }\end{array}$ & [85] \\
\hline GEM-Lip/GeIMA & MG63 & $\begin{array}{l}\text { It can achieve controlled release of GEM, can be used in anti-tumor } \\
\text { chemotherapy. }\end{array}$ & [86] \\
\hline GeIMA/PEGDA & MC3T3-E1 & $\begin{array}{l}\text { Improve structural stability and mechanical strength while promoting } \\
\text { cell proliferation and differentiation. }\end{array}$ & [87] \\
\hline BG/GelMA & hTMSCs & $\begin{array}{l}\text { It induced both osteogenic differentiation of hTMSCs and bio-active, with } \\
\text { hydroxyapatite forming on its surface. }\end{array}$ & [88] \\
\hline GelMA/AuNP & MSCs & $\begin{array}{l}\text { Due to high porosity and interconnectivity, CT imaging and reconstruction } \\
\text { are clearer and more convenient for clinical diagnosis. }\end{array}$ & [89] \\
\hline GelMANEGF & hMSCs & $\begin{array}{l}\text { Mimic the complex architecture of tissues and contain multiscale vasculature } \\
\text { networks with high structural stability. }\end{array}$ & [90] \\
\hline GelMA/NDs & hASCs & $\begin{array}{l}\text { Significantly increase the stiffness and promote calcium deposition of the } \\
\text { network encapsulated hASCs. }\end{array}$ & [91] \\
\hline GelMA/BG-5/5 & MC3T3 & $\begin{array}{l}\text { Promotes mineralization and new blood vessel formation while avoiding } \\
\text { resorption of newly formed bone. }\end{array}$ & [92] \\
\hline GelMA/DBM & $\mathrm{C} 2 \mathrm{C} 12$ & $\begin{array}{l}\text { It shows higher cell toxicity,low toxicity, greatest osteoinductive potential } \\
\text { with a higher percentage of new bone and bone marrow formation. }\end{array}$ & [93] \\
\hline GelMA microspheres & BMSCs & $\begin{array}{l}\text { This kind of microspheres can maintain the vitality of stem cells, promote } \\
\text { cell proliferation, enhance osteogenesis and mineralization. }\end{array}$ & [94] \\
\hline
\end{tabular}




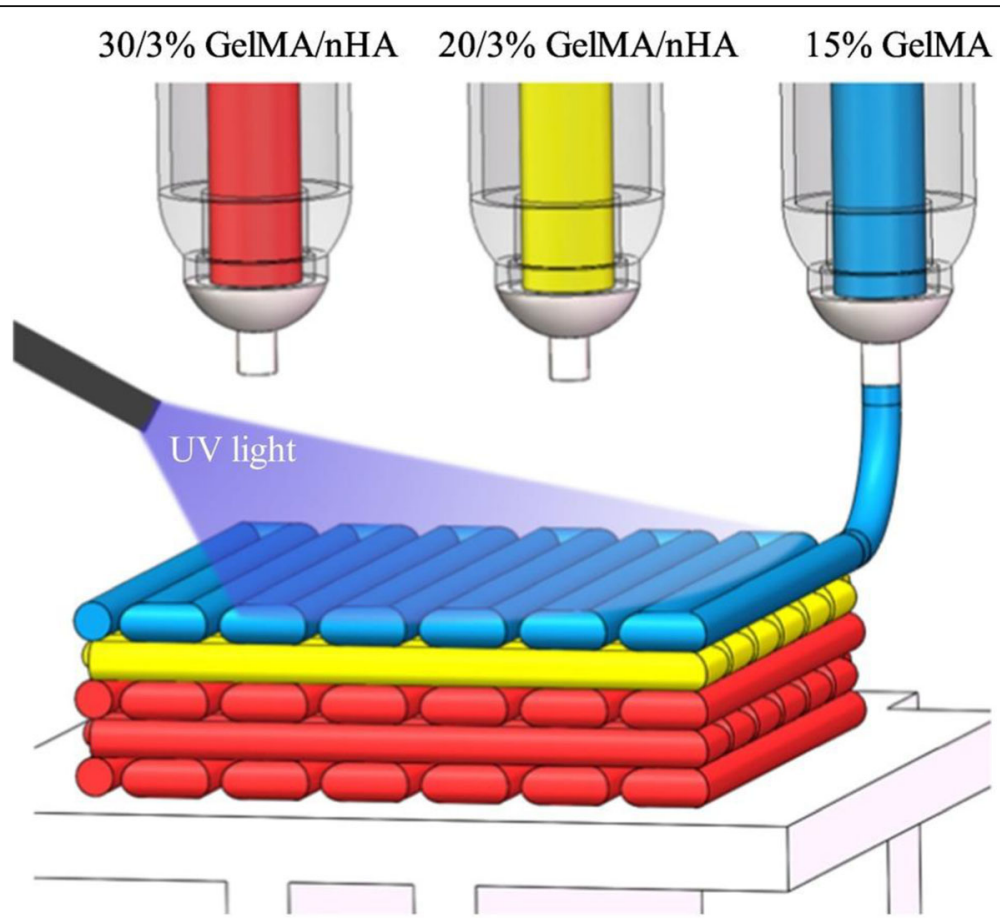

Fig. 2 Schematic diagram of preparation of multi-layered GeIMA/nHA scaffold. Reproduced with permission [71]. Copyright (2019), Elsevier Ltd.

diseases, such as fractures and bone cancer. However, the clinical application of this technology mainly faces two major problems, low retention and low engraftment of directly injected cells. In order to overcome the above problems, Zhao et al. [94] combined stem cell transplantation and microfluidics technology and successfully fabricated GelMA microspheres which offer controlled release of growth factors and contain BMSCs. From the result of histological analysis with H\&E and VG staining, they found that the GelMA microspheres delivering both BMSCs and BMP-2 together show the most extensive in vivo bone formation compared with the GelMA/BMSC sample, the GelMA sample, and the control sample.
Because the bones are the main weight-bearing organs of the body which are stretched by skeletal muscles. In the process of maintaining body posture and movement, the graft needs to have sufficient mechanical strength and toughness. How to make the hydrogel have stronger mechanical properties and toughness has become a major challenge in the application of bone defect repair. Gao et al. construct a novel scaffold, PACG-GelMA, with with a high tensile strength (up to $1.1 \mathrm{MPa}$ ), outstanding compressive strength (up to $12.4 \mathrm{MPa}$ ), large Young's modulus (up to $320 \mathrm{kPa}$ ), and high compression modulus (up to $837 \mathrm{kPa}$ ) [80], via 3D printing technology. Beside its outstanding mechanical property,it also
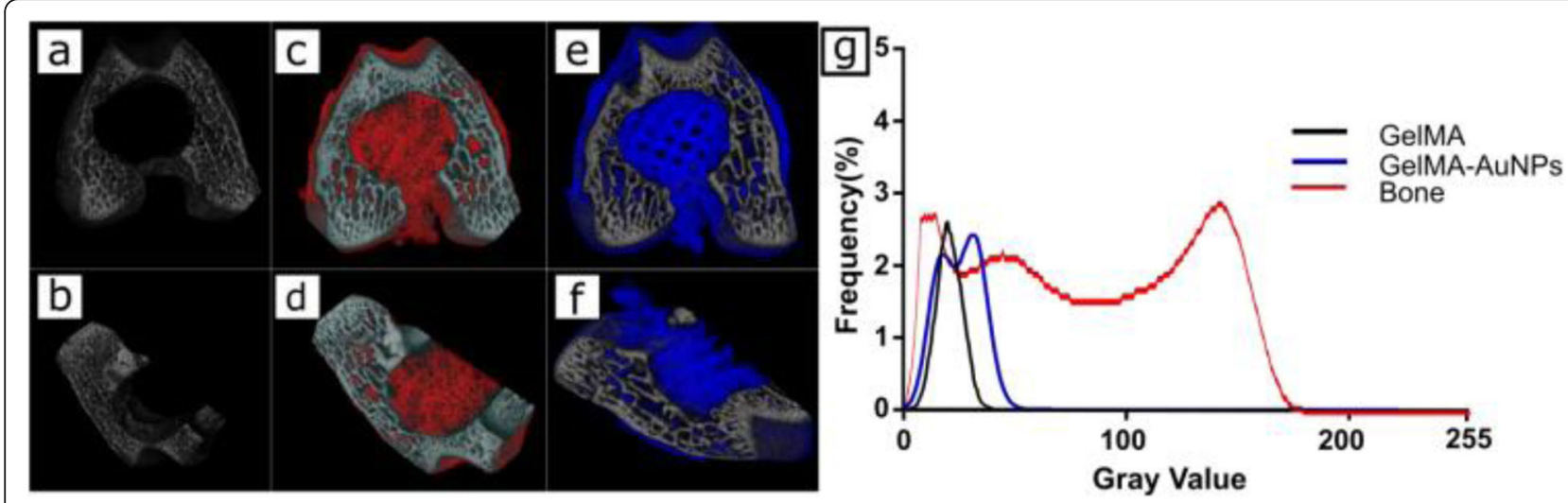

Fig. 3 CT tomogram of mouse bone injury area (a-f). Empty group(a-b), GelMA group(c-d), GelMA-AuNPs group(e-f). Comparison of the radiopacity of the three experimental groups(g). Reproduced with permission [89]. Copyright (2019), MDPI 


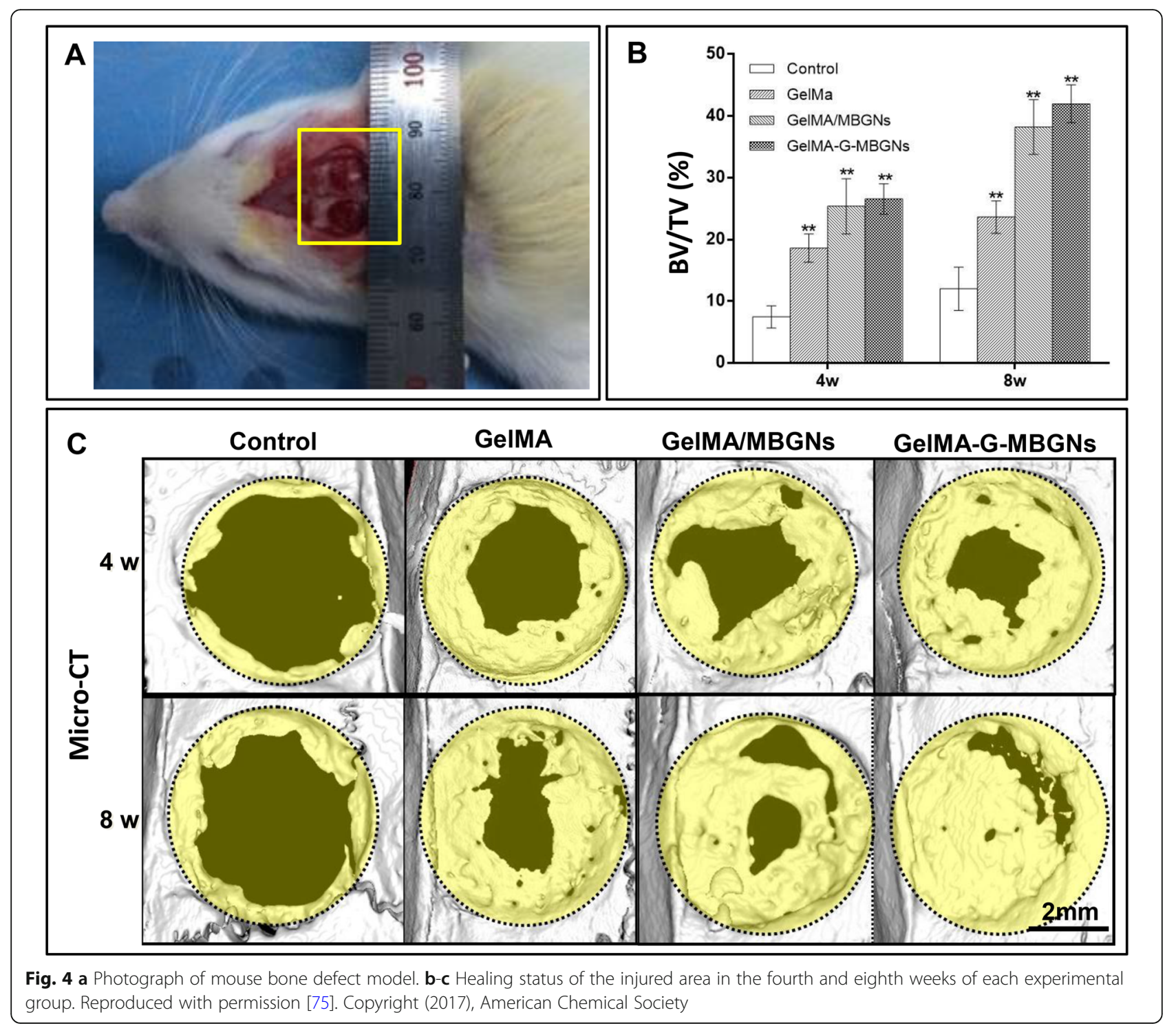

obtain tunable biodegradability by adjusting ACG/ GelMA ratios. The 12-week transplant experiment prove the PACG-GelMA significantly facilitates concurrent regeneration of cartilage and subchondral bone.

\section{GelMA for cartilage engineering}

The articular cartilage is a layer of shiny connective tissue covering the articular surface. The articular cartilage surface is elastic, has good lubrication, has a small friction coefficient, and absorbs shocks. The matrix in the cartilage is in a gel state and has greater toughness. Cartilage is a connective tissue absorbing the pressure between bone and bone or maintaining the appearance of the organ. The cartilage does not contain blood vessels and nutrients penetrate into the intercellular substance from the blood vessels in the periosteum to nourish bone cells, which led to the limited self-repair capacity.
Based on these, the treatment of cartilage defects is one of the major challenging clinical problems for clinical doctors and recent application of GelMA in the cartilage regeneration field and they are summarized in Table 4.

How to fabricate cartilage repair materials with 3D structure is a research hotspot in the field of tissue engineering. In recent years, new materials prepared by $3 \mathrm{D}$ printing technology have been widely used in the field of cartilage repair. Mouser et al. [97] discussed the influence of HAMA on the printing performance of GelMA / gellan and the cartilage forming ability of ACPCs and MSCs. They reported that HAMA can greatly enhance the stability of $3 \mathrm{D}$ printed structures without affecting the formation of cartilage, and found that ACPCs have higher cartilage formation potential than MSCs. Chung et al. [101] used the method of mixed bioprinting to fabricate a novel 3D scaffold where they used GelMA and 


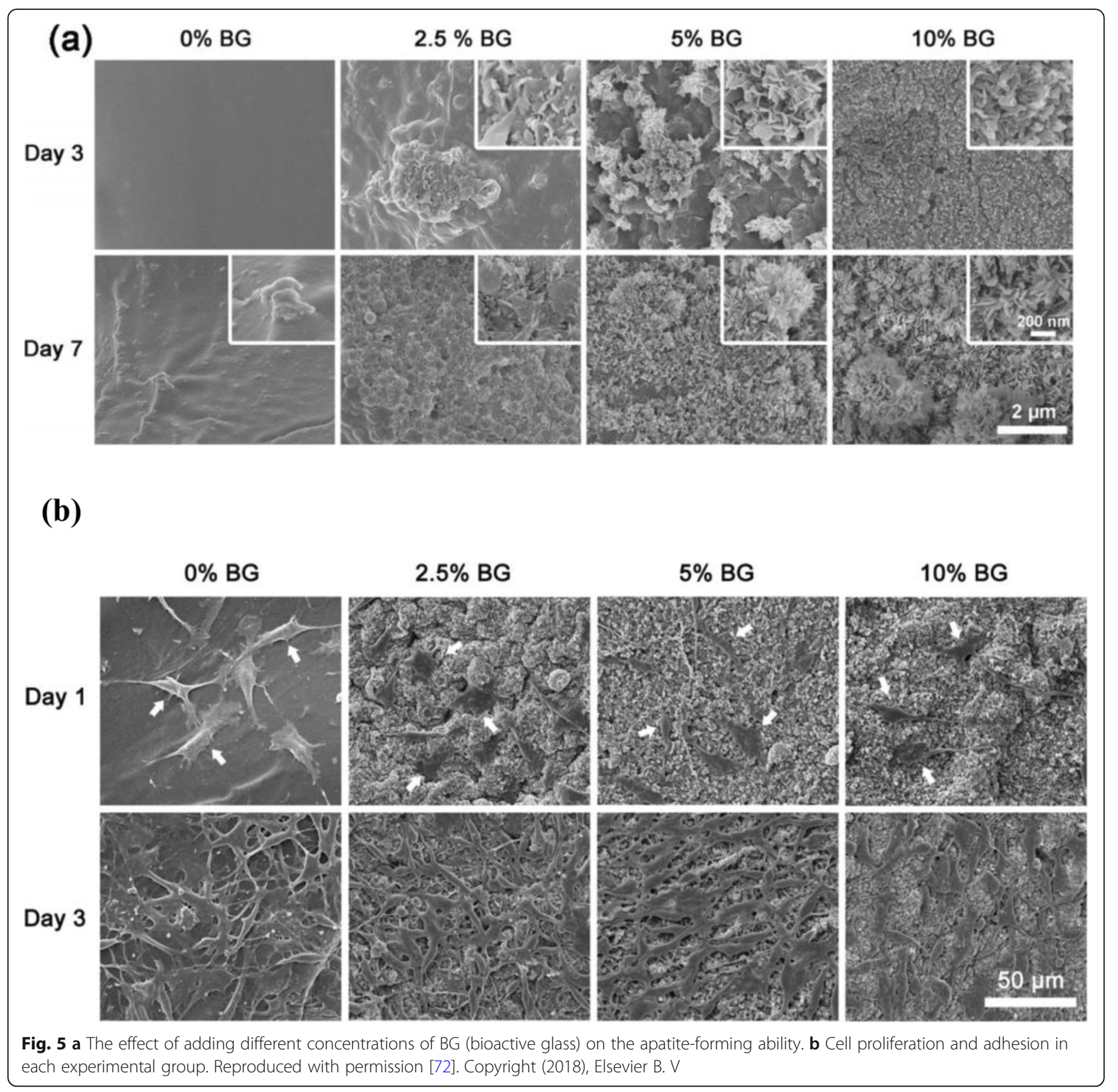

HAMA hydrogels as cell carriers, and added PCL to further enhance the strength of the material to ensure that the ear cartilage tissues can have sufficient mechanical properties. They concluded that the combined use of GelMA, HAMA, and PCL can promote and ensure the differentiation and proliferation of cells while ensuring that otic cartilage maintains morphological integrity. In order to make the chondrocytes encapsulated with hydrogel play the best performance in the repair of cartilage defects, Mouser et al. [96] designed a series of experiments to study the effect of the spatial distribution of chondrocytes on the process of cartilage repair. A 57- day culture experiment proves that the repair of cartilage tissue under conditions $\mathrm{C}$ and $\mathrm{D}$ (Fig. 6) is the best, regardless of the initial cell density.

Pahoff et al. [100] studied the effect of the source of gelatin and the type of photoinitiator on the ability of human articular chondrocytes in redifferentiating in GelMA / HAMA hydrogel. By comparing bovine (B) or porcine-derived (P) GelMA, two different sources of hydrogel, and lithium acylphosphinate (LAP) and visible light (405 nm) or Irgacure 2959 (IC) and UV light (365 $\mathrm{nm}$ ), two different types of photoinitiators, they found that the hydrogel constructs prepared with bovine- 
Table 4 The recent application of GelMA in cartilage engineering

\begin{tabular}{|c|c|c|c|}
\hline Polymer scaffold & Cell & Achievement & Reference \\
\hline GelMA-MeCS & Chondrocytes & $\begin{array}{l}\text { Can significantly stimulate cartilage tissue and promote the proliferation } \\
\text { and differentiation of chondrocytes. }\end{array}$ & [95] \\
\hline GelMA/gellan & Chondrocytes & $\begin{array}{l}\text { The effect of cell distribution on tissue defect repair was explored, and } \\
\text { it was concluded that tissue repair effect by encapsulating cells in } \\
\text { hydrogel and placing at the bottom of the wound was the best. }\end{array}$ & [96] \\
\hline GelMA/gellan/ HAMA & $\begin{array}{l}\text { ACPCs } \\
\text { MSCs }\end{array}$ & $\begin{array}{l}\text { Increased filament stability, highest chondrogenic potential was observed } \\
\text { for MSCs, followed by ACPCs. }\end{array}$ & [97] \\
\hline GelMA/PAM & Chondrocytes & Increase the structural stiffness of the material while having better biological functions. & [98] \\
\hline ODMA-GeIMA & BMSC & $\begin{array}{l}\text { Shows mechanical stability at body temperature and a sustained protein release with } \\
\text { good bio-compatibility and affinity for cells and tissues. }\end{array}$ & [99] \\
\hline GelMA & Chondrocytes & $\begin{array}{l}\text { The effects of different sources of GelMA and different types of light inducers on the } \\
\text { performance of hydrogels were compared, and it was concluded that using bovine- } \\
\text { derived GelMA and Irgacure can prepare hydrogels with the most similar characteristics } \\
\text { to articular cartilage. }\end{array}$ & [100] \\
\hline PCL /GelMA-HAMA & MSCs & It can provide optimal porosity and mechanical properties for cells to survive. & [101] \\
\hline GelMA and CS-AEMA & BM-MSCs & Shows high cell density, high cell viability and high printing resolution. & [102] \\
\hline GelMA-AGA & BMSCs & $\begin{array}{l}\text { Can promote the production of large amounts of type II collagen and repair } \\
\text { most of the defect areas(>95\%). }\end{array}$ & [103] \\
\hline GelMA & hBM-MPCs & Provide a new platform for studying bone and muscle growth and development. & [104] \\
\hline PEGDA/GelMA & MSCs & $\begin{array}{l}\text { Increases the resolution of printing while accelerating the proliferation and differentiation } \\
\text { of the loaded cells. }\end{array}$ & [105] \\
\hline
\end{tabular}

derived GelMA and Irgacure 2959 and photocrosslinking at $365 \mathrm{~nm}$ showed the most similar characteristics to natural articular cartilage.

\section{GelMA for skeletal muscle engineering}

Skeletal muscles, which are composed of many muscle fibers bounded together by epimysium [112], play an important role in the body's various sports and posture maintenance. The human body has more than six hundred skeletal muscles which are about $40 \%$ of the total body weight. Once the muscle tissue is pulled or broken, it will cause unbearable pain and dyskinesia of related tissues. The purpose of muscle tissue engineering is to culture muscle tissue in vitro and transplant the cultured tissue into the patient to treat muscle defect. The recent applications are summarized in Table 5 .

Although several materials have been applied to muscle tissue engineering, how to better imitate the structure and function of natural muscle tissue is still a huge challenge. Three-dimensional (3D) bioprinting is a powerful weapon to deal with the problem that how to mimic the hierarchical structure of native tissues. Andrea et al. [107] made a full comparison of three photocrosslinkable composite materials (GelMA/AlgMA, GelMA/CMCMA and GelMA-PEGDA), which are often used as bio-inks for 3D bio-printing,in the aspect of stiffness, cell behavior, cell differentiation et al. They concluded that compared with GelMA-PEGDA, GelMA/ AlgMA and GelMA/CMCMA shows higher cell proliferation, viability and can be used to print non-biodegradable skeletal muscle structures. Because carbon nanotubes (CNTs) can significantly improve the mechanical and electrical properties of GelMA, many researchers have explored the application of CNT/ GelMA in the field of tissue engineering. Since muscle fibers grow vertically in their natural state, GelMA hydrogels containing randomly distributed CNTs can not provided optimal environment for growth of muscle fibers and most of the unique properties of CNTs are exerted in the direction of the tube axis [113]. Ahadian et al. [109]

\begin{tabular}{|l}
\hline $\begin{array}{l}\text { \# of cells/ml } \\
\text { Hydrogel: } \\
\text { Defect bottom: } \\
\text { Fig. } \mathbf{6} \text { Schematic cross-sectional overview of the different Chondrocyte filled conditions. Reproduced with permission [96]. Copyright (2018), } \\
\text { Springer Spektrum }\end{array}$ \\
$20 \cdot 10^{6}$
\end{tabular}


Table $\mathbf{5}$ The recent application of GelMA in skeletal muscle engineering

\begin{tabular}{|c|c|c|c|}
\hline Polymer scaffold & Cell & Achievement & Reference \\
\hline GeIMA-PdMGSMW & $\begin{array}{l}\mathrm{C} 2 \mathrm{C} 12 \\
\mathrm{NIH} 3 \mathrm{T3}\end{array}$ & $\begin{array}{l}\text { Improve the mechanical properties and electrical conductivity of the } \\
\text { material while accelerating C2C12 cells proliferation and muscle repair. }\end{array}$ & [106] \\
\hline GelMA/CMCMA GelMA/AlgMA & $\mathrm{C} 2 \mathrm{C} 12$ & Show high cell viability, myogenesis, cell proliferation and structural stability. & [107] \\
\hline GelMA/Alg & $\mathrm{C} 2 \mathrm{C} 12$ & Explored and reported the optimal concentration of GelMA that promotes muscle formation. & [108] \\
\hline GelMA-CNT & $\mathrm{C} 2 \mathrm{C} 12$ & $\begin{array}{l}\text { Show superior conductivity and mechanical properties and increase the myogenic } \\
\text { gene and protein expression. }\end{array}$ & [109] \\
\hline GelMA & $\begin{array}{l}\mathrm{C} 2 \mathrm{C} 12 \\
\mathrm{PC} 12\end{array}$ & $\begin{array}{l}\text { Improve differentiation Myotube alignment, length and coverage area with } \\
\text { enhanced myotube formation. }\end{array}$ & [110] \\
\hline GelMA & $\mathrm{C} 2 \mathrm{C} 12$ & $\begin{array}{l}\text { Accelerate the process of } \mathrm{C} 2 \mathrm{C} 12 \text { cells forming myotubes and increase the } \\
\text { expression of related genes and proteins. }\end{array}$ & [111] \\
\hline
\end{tabular}

used dielectrophoresis (DEP) to vertically align carbon nanotubes within GelMA hydrogels (Fig. 7), so that skeletal muscle cells seeded on these hydrogels could only attach to and sense CNTs in their radial direction,which can better promote the growth of muscle fibers compared with randomly distributed CNTs and horizontally aligned CNTs.

\section{GelMA for heart engineering}

In recent years, with the acceleration of life pace (result in irregular work schedule and unhealthy diet) and the aging of the population, more and more people are suffering from heart disease, and the number of deaths due to heart disease is also increasing year by year. Infection, heart tissue defects, and insufficient blood and oxygen supply to the heart are several major factors that cause heart disease. Tissue engineering aims to use artificial objects to repair damaged tissue or provide more suitable growth conditions for cells or tissue. At present, many researchers have developed a variety of new materials for clinical use based on the excellent performance of GelMA. The recent applications of GelMA in heart engineering are summarized in Table 6.

For most cells, sufficient oxygen is the basic guarantee for their survival, and cardiomyocytes are no exception. In many cases of heart disease, such as myocardial infarction or cardiac embolism, long-term ischemia and hypoxia can trigger a stress response of the heart tissue and cause irreversible damage and necrosis of the heart tissue. In order to solve the problem of cell hypoxia, Alemdar et al. [116] combine calcium peroxide (CPO) with GelMA (Fig. 8) to provide a new method for treating myocardial hypoxia-type diseases. The CPO/GelMA was proved to have the ability to release a large amount of oxygen and can effectively reduce the death of cells under hypoxic conditions (Fig. 9).

How to maintain the integrity and continuity between peripheral heart muscle cells is a major challenge in the repair of heart tissue. CNT (carbon nanotube)/GelMA has been proven to have more suitable mechanical and electrophysiological properties, it can protect myocardial cells and improve their beating rate while providing cells

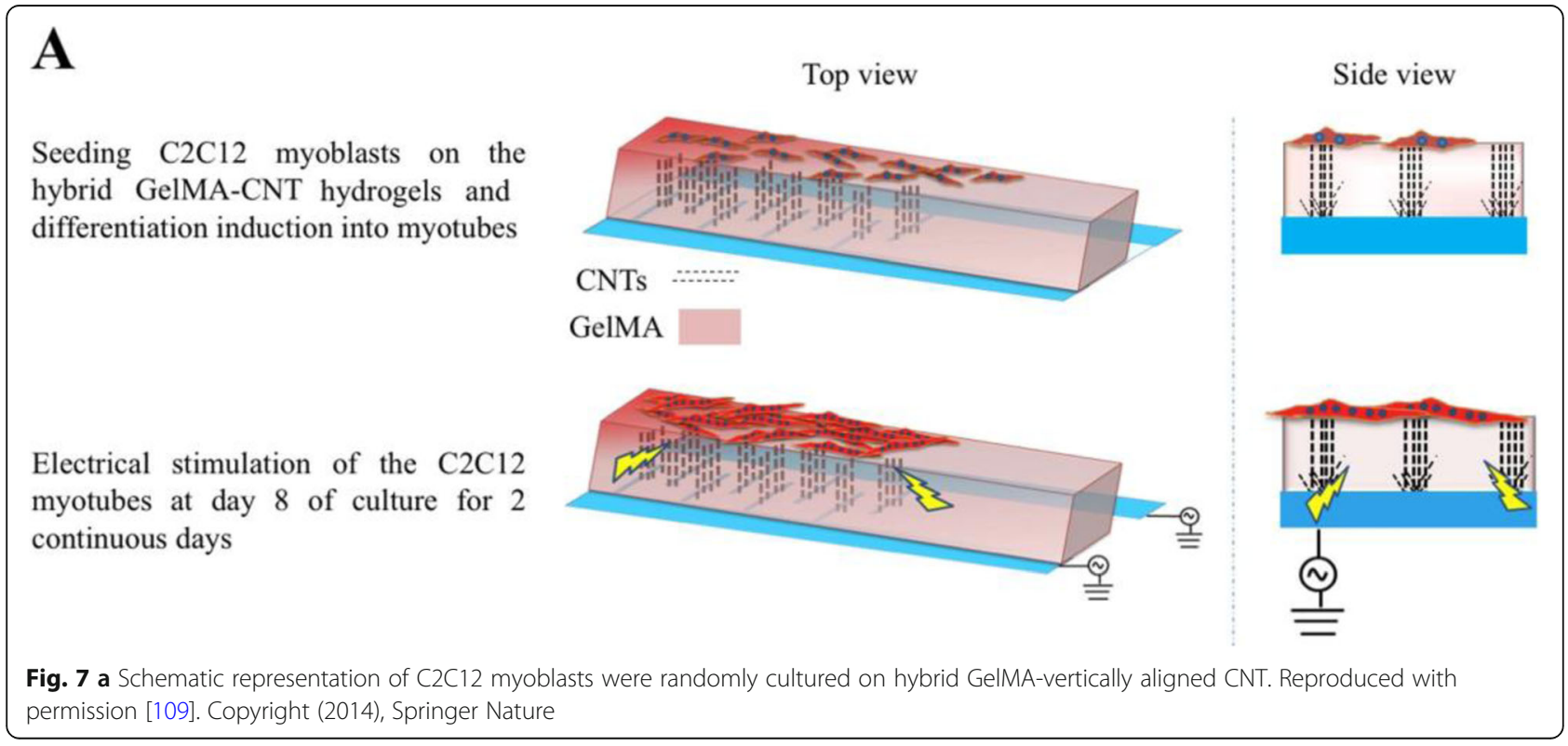


Table 6 The recent application of GelMA in heart engineering

\begin{tabular}{|c|c|c|c|}
\hline Polymer scaffold & Cell & Achievement & Reference \\
\hline CNT/GeIMA & NRVMs & $\begin{array}{l}\text { It can better imitate the electrophysiological properties of } \\
\text { cardiomyocytes and provide the best living environment for } \\
\text { newborn cells while protecting the original cells. }\end{array}$ & {$[114]$} \\
\hline GelMA & hASCs & $\begin{array}{l}\text { By up-regulating the expression of various genes in hACSs, the } \\
\text { blood supply and function of the infarct area can be effectively restored. }\end{array}$ & [115] \\
\hline CPO-GelMA & CSPS & $\begin{array}{l}\text { By releasing a large amount of oxygen can effectively reduce the } \\
\text { death of cells under hypoxic conditions. }\end{array}$ & [116] \\
\hline GelMA & hiPSCs & $\begin{array}{l}\text { Shows very high hiPSCs viability and the new tissue can effectively } \\
\text { simulate the spontaneous contraction of cardiomyocyte. }\end{array}$ & [117] \\
\hline rGO/PEGDA/GelMA & Rat Cardiomyocytes & $\begin{array}{l}\text { It was elastic and sensitive to cellular behavior, which could convert } \\
\text { biological signals into a visual signal. }\end{array}$ & [118] \\
\hline GeIMA/ cECM & $\mathrm{hCPCs}$ & $\begin{array}{l}\text { Show homogeneous distribution of cells and matrix and improve } \\
\text { differentiation and angiogenic potential. }\end{array}$ & [119] \\
\hline GelMA/Bio-IL & $\begin{array}{l}\text { Primary cardiomyocytes } \\
\text { and cardiac fibroblasts }\end{array}$ & $\begin{array}{l}\text { Have optimal mechanical, electrophysiological and spontaneous } \\
\text { contraction properties which are very similar to natural cardiomyocytes. }\end{array}$ & [120] \\
\hline GelMA-Ppy/ ES-GelMA/PCL & CMs & $\begin{array}{l}\text { Enhance the function of cardiomyocytes (CMs) and yield their synchronous } \\
\text { contraction. Enhance cardiac function and revascularization. }\end{array}$ & [121] \\
\hline
\end{tabular}

with a suitable proliferation environment [122]. Hongyu Sun et al. further improved the research on the structural integrity of engineering cardiac tissues (ECTs)(Fig. 10). They reported three important discoverys: (i) the incorporation of CNTs enhances ID assembly and formation in engineered cardiac tissues; (ii) b1-integrin mediated FAK signaling pathway plays a critical role in the CNT-induced gap junction formation; (iii) b1integrn mediated Rho pathway is responsible for the CNT-induced mechanical junction formation [114].

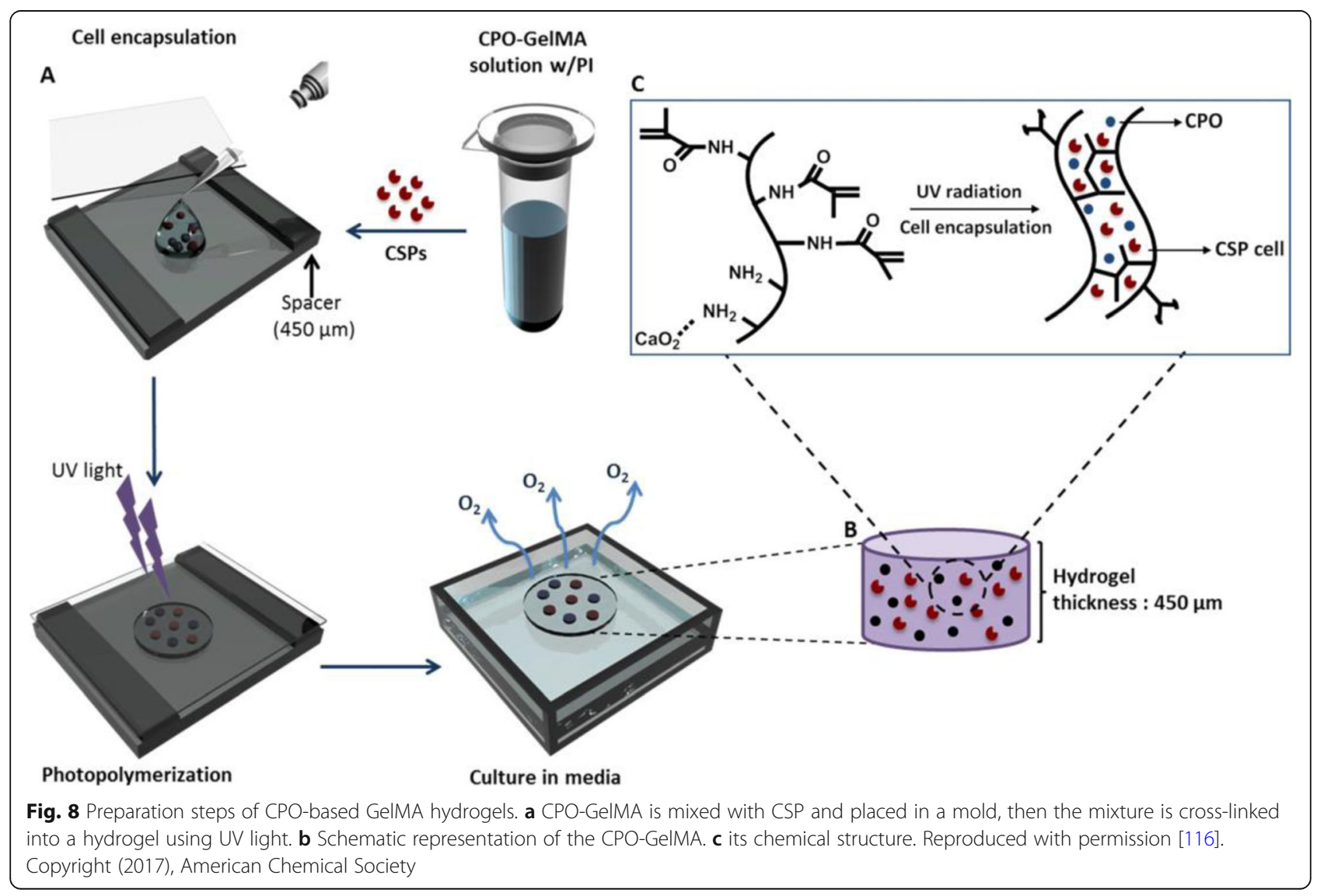




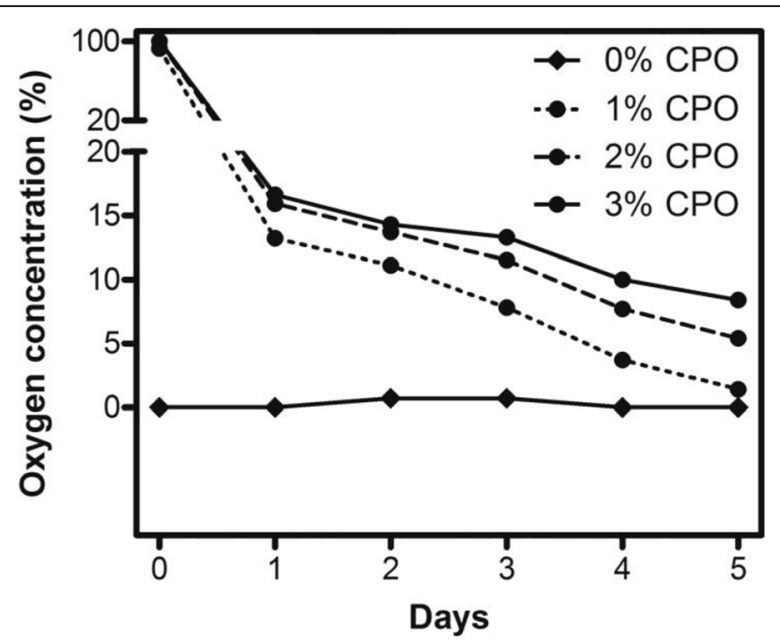

Fig. 9 Comparison of oxygen release capacity of various groups of hydrogels under hypoxia conditions. Reproduced with permission [116]. Copyright (2017), American Chemical Society

After the myocardial tissue is necrotic, the body will repair itself, eventually the necrotic area will be replaced by fibrous scar tissue, which greatly affects the original contractile function of the heart, resulting in a decrease in the cardiac ejection fraction. Brian W. Walker et al. have fabricated a novel scaffold (GelMA/Bio-IL), which can form ionic bonds with the surrounding myocardial tissue so that it can firmly adhere to the surrounding tissue without stitching [120]. Judging from the results of gap junction protein connexin 43 expression, this novel scaffold not only can promote the growth of primary cardiomyocytes but also obtain optimal mechanical and conductive properties, which are similar to the native myocardium.

\section{GelMA for skin tissue engineering}

As the body's first non-specific immune barrier against external pathogens, skin plays an extremely important role in maintaining the stability of the body's environment. Small skin damage, the body can heal itself quickly with mild inflammation. However, large areas of skin damage, if not treated clinically promptly, can lead to a severe immune response and eventually death. At present, a common problem with the use of skin substitutes in clinical treatment is that the graft can not be effectively adhered to the surface of defect tissue, and the slow angiogenesis rate easily leads to the local necrosis of the graft. Moreover, GelMA could be seeded on the surface of the wound, fill the wound (no matter what shape it is) and crosslink in situ within minutes or even seconds following UV light exposure. GelMA, which has tunable mechanical properties, degradation rates, good bio-compatibility and can effectively promote the growth and differentiation and stratification of epithelial cells, is considered to be a potential skin substitute. The recent applications of GelMA in epidermal tissue engineering are summarized in Table 7.

Vascular is responsible for the task of transporting nutrients and taking away excretion waste. Restoring blood supply in damaged areas as soon as possible while repairing skin tissue can greatly reduce the risk of necrosis of the graft. Especially when diabetes is combined with vascular lesions, the wound is extremely difficult to heal. The capillaries can be established as soon as possible plays a decisive role in the rapid healing of the wound. In order to solve this problem, Chen et al. [127] devised a desferrioxamine (DFO)-loaded GelMA hydrogel for accelerating the development of the vascular network and skin reconstruction (Fig. 11). The hydrogel was seeded on the wound surface and exposed to UV light $\left(6.9 \mathrm{~mW} \mathrm{~cm}^{-2}\right)$ in the presence of Irgacure 2959 . Then the wound area was covered with a volume of $3 \mathrm{M}$ Tegaderm and a sterilized piece of gauze was placed over the dorsum afterwards. The results showed that at the fourth hour, the experimental group had significantly more vascular tubule formation of HUVECs than the control group, and the blood vessels formed at the twelfth hour become thicker. Sun et al. [132] fabricated a GelMA scaffold by electrospinning technology to deal with the distal necrosis of random skin flap. Through Live/Dead analysis and CCK-8 assay, this scaffold showed good cell compatibility. In the 7-day flap vitality experiment, the GelMA group shows lower necrosis ratio, inflammatory response and higher blood perfusion, compared with control group and gelatin group.

Bakhsheshi-Rad et al. used GelMA/HAMA as a carrier for loading ADSC, which also makes good progress in promoting angiogenesis (Fig. 12) and wound healing. They verified the effect of this material on promoting angiogenesis and skin wound healing by chick chorioallantoic membrane (CAM) assay. We can clearly find the total number and length of blood vessels growing on the CAM tissue adjacent to the VEGF loaded and ADSC loaded hydrogel was significantly higher compared to unloaded ones [124].

Get rid of the mindset of using GelMA as a platform for single cell culture, Zhang et al. [126] utilized the GelMA as a platform for co-culture of uc-MSCs and HUVECs and studied the effect of regulating the different ratios of the two cells on epidermal regeneration. The result reveals that an uc-MSC:HUVEC ratio of 50: 50 demon resulted in the highest cell proliferation and expression of angiogenic markers.

\section{GelMA for cornea stroma and other tissue engineering}

According to the WHO report [138], more than 10 million people lose their eyesight due to corneal diseases 


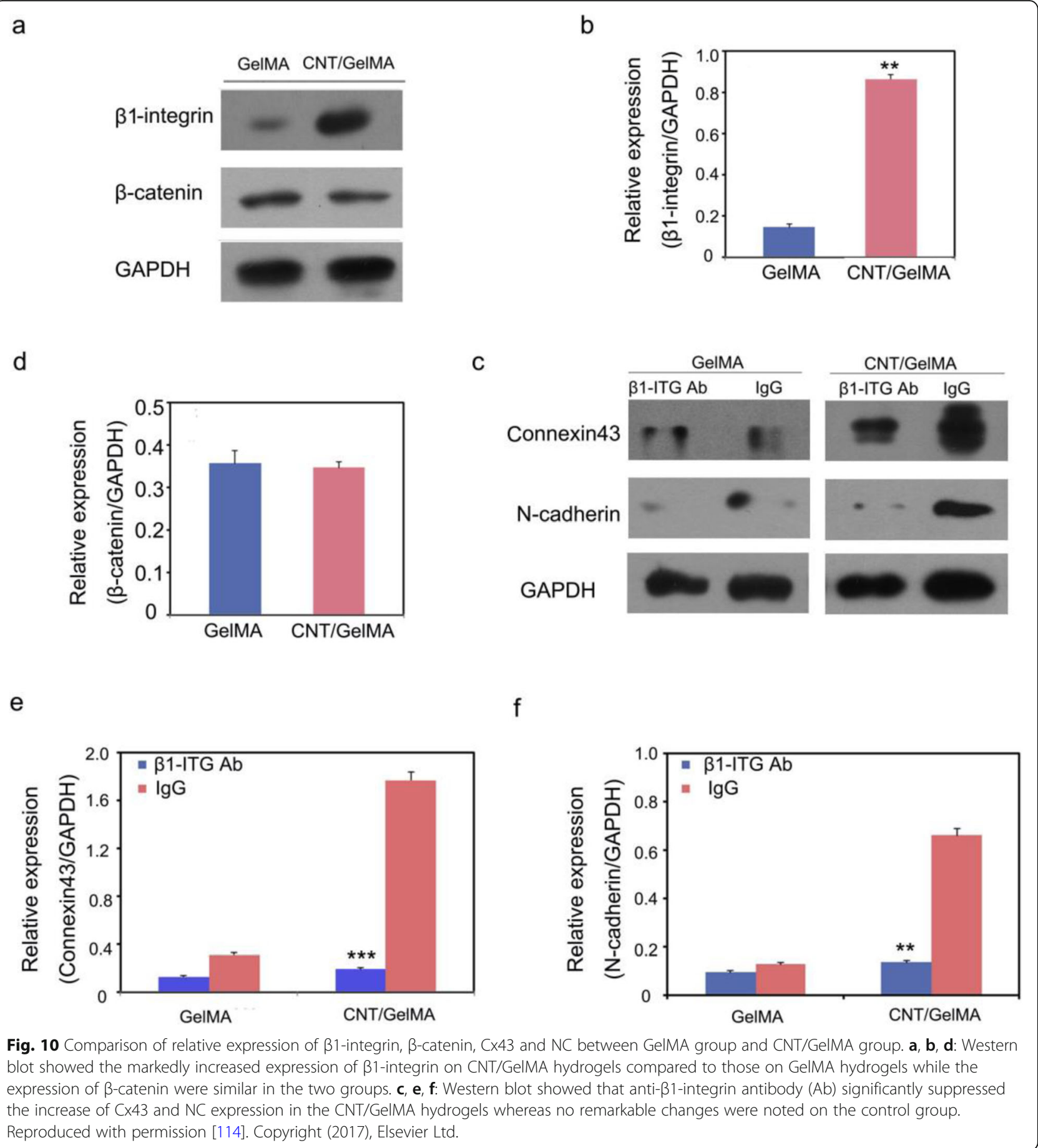

every year, which is a huge blow to the lives of patients. At present, there are the following methods for clinical treatment of such diseases:penetrating keratoplasty, artificial keratoprostheses and decellularized corneal tissue. However the above methods all have more or less disadvantages,such as poor mechanical and optical properties, the shortage of donor corneal tissue. In recent years, the application of GelMA in the field of corneal repair has also received widespread attention because GelMA can obtain appropriate mechanical properties and optical properties through photocrosslinking, and its RGD amino acid sequence has been shown to maintain $98 \%$ survival rate of keratocyte during 21 days of culture [139]. GelMA is not only widely researched in the above 
Table 7 The recent application of GelMA in skin tissue engineering

\begin{tabular}{|c|c|c|c|}
\hline Polymer scaffold & Cell & Achievement & Reference \\
\hline PCL/GeIMA-CEX & / & $\begin{array}{l}\text { Have a broad-spectrum antibacterial effect of minimizing the chance } \\
\text { of infection during wound healing. }\end{array}$ & [123] \\
\hline GelMA/HAMA & ADSC & $\begin{array}{l}\text { It can further promote the formation of blood vessels in the injured area, } \\
\text { and the transparent material is more convenient for clinical observation. }\end{array}$ & [124] \\
\hline TA-GelMA & Dermal fibroblast cells & $\begin{array}{l}\text { It has good bio-compatibility and has greatly improved various } \\
\text { mechanical properties. }\end{array}$ & [125] \\
\hline GelMA & $\begin{array}{l}\text { UC-MSCS } \\
\text { HUVECS }\end{array}$ & $\begin{array}{l}\text { Promote cell proliferation and differentiation by increasing related gene } \\
\text { expression. }\end{array}$ & [126] \\
\hline DFO-Gelma & / & Accelerate blood supply re-establishment and wound healing. & [127] \\
\hline MeTro/GelMA-Tet213 & / & $\begin{array}{l}\text { Better mechanical properties, in vitro and in vivo degradation, swellability, } \\
\text { and porosity }\end{array}$ & [128] \\
\hline GelMA & Fibroblasts & $\begin{array}{l}\text { Accelerate wound healing by providing a suitable environment for cell } \\
\text { proliferation and adhesion. }\end{array}$ & [129] \\
\hline GeIMA/anti-IL-6 & / & $\begin{array}{l}\text { It can effectively reduce the inflammatory response and accelerate } \\
\text { wound healing. }\end{array}$ & [130] \\
\hline GelMA/Col-Ty & $\begin{array}{l}\text { HEM } \\
\text { HaCat } \\
\text { HDF }\end{array}$ & $\begin{array}{l}\text { Promote wound healing by promoting the proliferation and } \\
\text { differentiation of HEM without affecting HaCat. }\end{array}$ & [131] \\
\hline GelMA electrospun fibers & $\begin{array}{l}\text { HDF } \\
\text { HUVECS }\end{array}$ & $\begin{array}{l}\text { GelMA electrospun fibers can accelerate the formation of vascular } \\
\text { network, and can promote cell adhesion and proliferation. Compared } \\
\text { with nanofibrous, this scaffold not only can promote skin flap } \\
\text { regeneration but also reduce the level of inflammation. }\end{array}$ & [132] \\
\hline
\end{tabular}

fields, but also has outstanding performance in other tissue engineering and these applications are summarized in the Table 8 .

The decellularization cornea applied in cornea stroma engineering can not mimic the environment of extracellular matrix well because it changes the original ECM micro-environment during the decellularization process. In order to solve this problem Uyaniklar et al. [133] fabricate a novel scaffold which is a combination of GelMA and decellularized matrix. They reported that this material has better compressive modulus (5040 and $870 \mathrm{kPa}$ ) and light transmittance value (53.6\%), due to the special structure of GelMA makes up for the inherent shortcomings of the decellularization matrix. Bektas et al. [134] used $3 \mathrm{D}$ printing technology to prepare a three-dimensional scaffold (Fig. 13) with higher stability in PBS, better ability of ensuring cell viability and 2-fold mechanical properties compared with native cornea.

To further prove the real role in corneal repair of the GelMA hydrogels in vivo, Bektas et al. [139] carried out a in vivo study using eyes of two white New Zealand rabbits. Observation after transplantation for up to 8 weeks, the result showed that no inflammation and immune rejection were observed and the hydrogels heal well with the host tissue. Kong et al. [28] utilized fiber (PECL) to reinforce GelMA hydrogel and made a comparation among 3D fiber hydrogel, 3D GelMA hydrogel, and $2 \mathrm{D}$ culture dish and finally proved fiber reinforced
PECL/ GelMA can provide an optimal micro-environment for promoting keratocyte guided differentiation and the regeneration of damaged corneal stroma.

Cui et al. [136] reported the application of GelMA in liver tissue engineering. They used GelMA hydrogel as a matrix to construct 3D lobule-like tissues for co-culture of hepatocytes and fibroblasts. Firstly, they fabricate hepatocytes encapsulated micromodules through microfluidic technology. Then, assemble cellular micromodules through non-contact pick-up strategy. At last the assembled micromodules were coated with fibroblast-laden GelMA. They reported the 3D lobule-like microtissues can maintain $90 \%$ cell viability and improve hepatic cell albumin secretion.

Diseases of the oral mucosa, such as oral ulcers, can cause severe pain in the damaged area, and long-term wound non-healing can increase the chance of cancer in the damaged area. Zhang et al. [137] fabricated an ideal soft tissue substitute for the repair of oral mucosal defects, by combining decellularized human amniotic particles (dHAP) and GelMA. They reported that GelMAdHAP not only can serve as a soft tissue replacement for oral mucosa repair, but also can stimulate rapid angiogenesis in the defect area.

\section{Conclusion and outlook}

In this article we mainly reviewed the recent development and applications of GelMA in the field of biomedicine. Based on its unique $3 \mathrm{D}$ structure (can mimic 


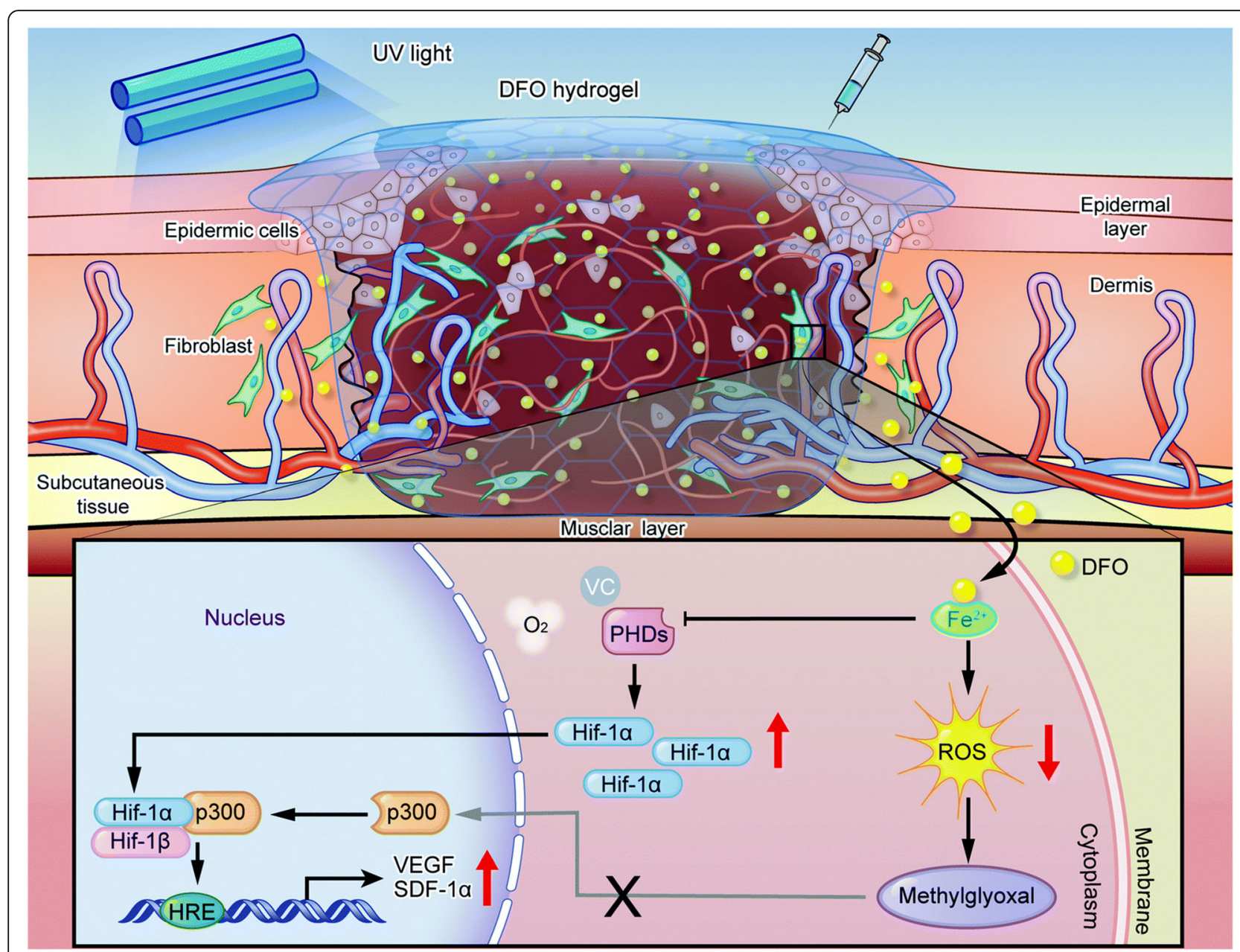

Fig. 11 Schematic diagram of DFO-GelMA hydrogels promoting wound healing by regulating HIF-1a signaling pathways. Reproduced with permission [127]. Copyright (2016), The Royal Society of Chemistry

ECM), RGD peptide sequence (can promote cell adhesion and proliferation), MMP degradable motifs and good biocompatibility (can be combined with other material to adjust related properties), GelMA has shown great potential in the fields of biomedicine and tissue engineering and has been widely used in these fields.

There are many advanced technologies that have been used to modify the relevant properties of GelMA so that it can have excellent performance in different biological fields. Many researchers have strengthened the mechanical properties of GelMA to make it suitable for 3D printing. Using GelMA as a bio-ink, via 3D printing technology, can prepare personalized scaffolds that can meet the needs of various tissue engineering. Through research, we found that applying electrospinning technology to the preparation of biomaterials based on GelMA can give the material excellent performance,such as high surface-to-volume ratio, tunable pore size (from from several to tens of micrometers) and high porosity (>90\%). Combining electrospinning technology with
GelMA can further improve GelMA's performance in tissue engineering, especially in promoting bone tissue regeneration and angiogenesis. Researchers have also used microfluidic technology to fabricate GelMA hydrogel microspheres. Through this technique, microspheres containing relevant tissue factors or cells can be directly obtained, and can be directly injected into the injury area to promote tissue repair.

However, the current research on GelMA is still insufficient and we believe that GelMA is worthy of further study in the following areas.

(1) Expanding material sources of GelMA and studying the effects of different animal skin sources on GelMA performance to further explore its greater potential in the biomedical field.

(2) Whether the modified GelMA, via physical or chemical method, will cause an immune response in the early stage of implantation or by its degradation products, and whether the degradation 


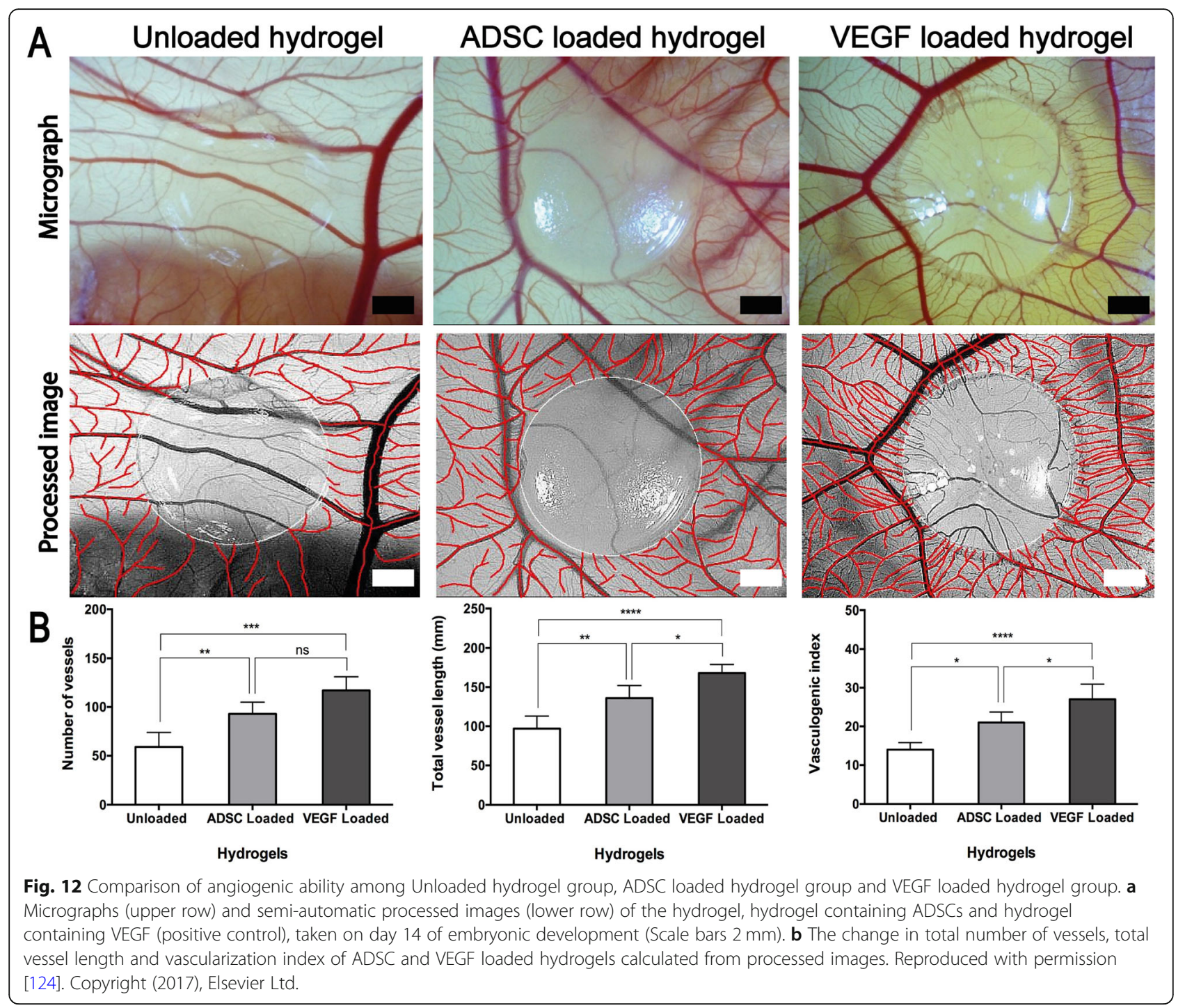

Table 8 The recent application of GelMA in cornea stroma and other tissue engineering

\begin{tabular}{|c|c|c|c|c|}
\hline Tissue & Polymer scaffold & Cell & Achievement & Reference \\
\hline \multirow[t]{4}{*}{ cornea stroma } & GelMA/ decellularized cornea & Keratocyte & $\begin{array}{l}10 \text { fold light transmittance value and } 6 \text { fold compressive } \\
\text { modulus compared to decellularized cornea }\end{array}$ & [133] \\
\hline & GelMA & HKs & $\begin{array}{l}\text { With good biocompatibility and suitable mechanical properties, } \\
\text { it can be well integrated with the host tissue without causing } \\
\text { serious immune rejection }\end{array}$ & [134] \\
\hline & PECL/ GelMA & LSSCS & $\begin{array}{l}\text { It has the light transmission and mechanical strength most } \\
\text { similar to the natural cornea, which can provide the best } \\
\text { repair and regeneration environment for the damaged stroma. }\end{array}$ & [28] \\
\hline & GelMA & Keratocyte & Allow better vision than nontransparent substrates. & [135] \\
\hline Liver & GelMA & $\begin{array}{l}\text { HepG2 } \\
\mathrm{NIH}-3 \mathrm{T3}\end{array}$ & $\begin{array}{l}\text { Maintained over } 90 \% \text { cell viability. The liver function of albumin } \\
\text { secretion was enhanced }\end{array}$ & [136] \\
\hline Oral mucosa & GelMA/dHAP & Human fibroblast HFF & $\begin{array}{l}\text { Improve mechanical properties and can promote repair of oral } \\
\text { mucosal defects within two weeks. }\end{array}$ & [137] \\
\hline
\end{tabular}




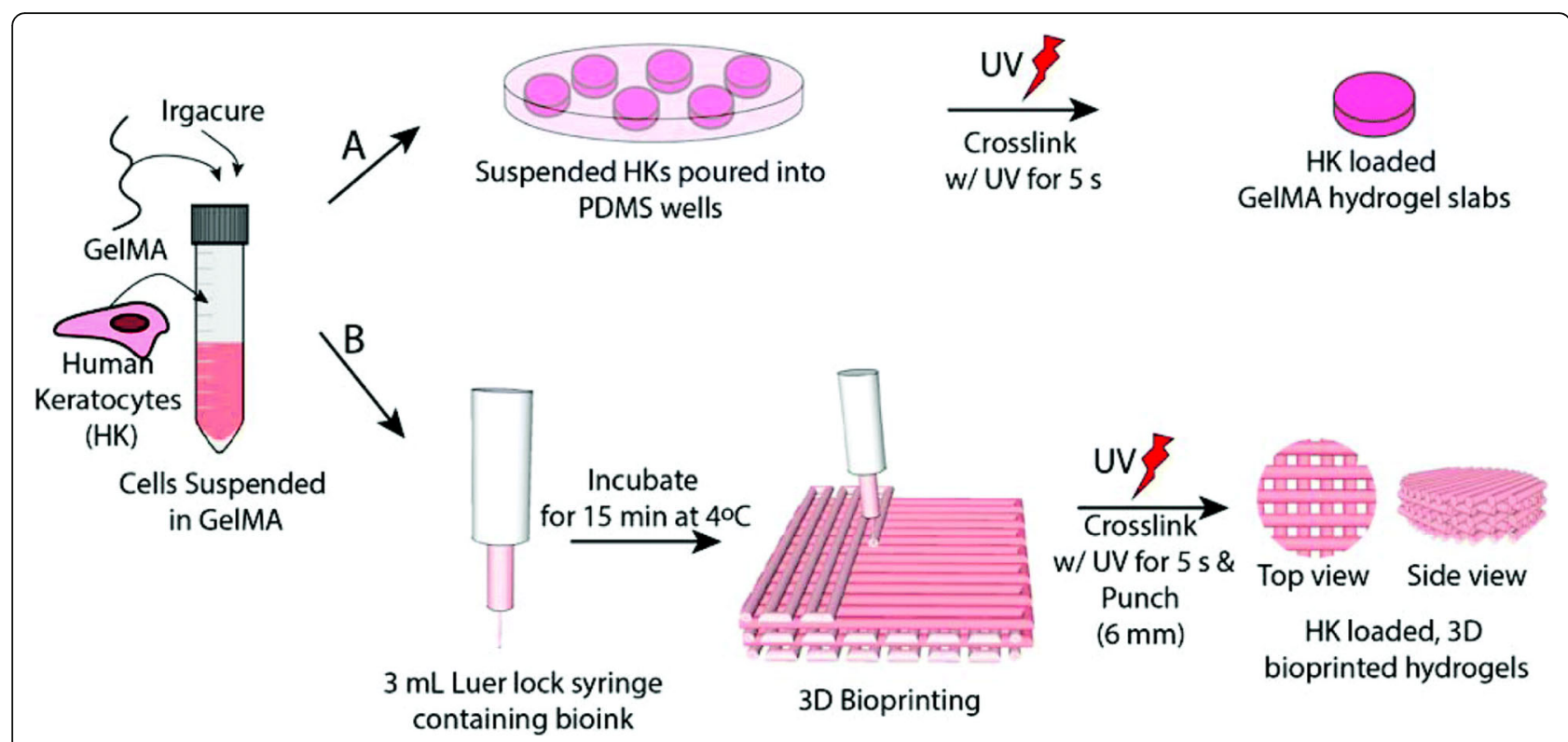

Fig. 13 Schematic diagram of preparation of HK loaded GelMA hydrogel slabs and 3D bioprinted hydrogels. Reproduced with permission [134]. Copyright (2019), The Royal Society of Chemistry

of GelMA will cause changes in hemodynamics and increase the risk of thrombosis are worthy of further study.

(3) How to ensure the preparation of large-scale GelMA hydrogels that meet clinical needs while ensuring the stability of their related traits is still a big challenge.

(4) In the field of promoting bone regeneration, the molecular mechanism and the signal path of modified GelMA to promote mineralization and new bone formation and mechanism of coordinated repair of blood supply, nerve and defect tissue need to be further clarified. GelMA was also widely used to repair some special tissues such as the heart, cornea and nerves, the mechanism of inducing the directional differentiation of cells to reduce the formation of scar tissues and restore the original functions of tissues and organs needs further investigation.

(5) Develop some new biomaterials with various composite functions based on GelMA, for example, temperature response, ion response, shape memory, magnetic response and develop the smart drug carrier based on GelMA via combining with other nanomaterials or micro-robots, which can realize the release of drugs at specific sites while prolonging the release time of drugs in vivo.

(6) From the perspective of bionics and the natural phenomena, develop some special functional structures based on GelMA, such as the vessel structure inspired by curled apple peel [140], for further expanding the application of GelMA in the field of biomedicine.

\section{Abbreviations}

AM: Acrylamide; ALN: Alendronate; AuNP: Gold nanoparticles; ADSCs: Adipose derived stem cells; AlgMA: Alginate-methacrylate; ACPCs: Articular cartilage progenitor cells; AGA: Acryloyl glucosamine; ALG: Alginate; BG: Bioactive glass; BMP-2: Recombinant Human Bone Morphogenetic protein-2; BMSCs: Bone marrow mesenchymal stem cells; $\beta$ TCP: $\beta$-tricalcium phosphate; Bio-IL: Bio-ionic liquid; CPT: Camptothecin; CBMA: Carboxybetaine methacrylate; CNT: Carbon nanotube; CPO: Calcium peroxide; CSPs: Cardiac side population cells; CECM: Cardiac extracellular matrix; CMs: Cardiomyocytes; CEX: Cephalexin; Col-Ty: Collagen/tyrosinase; CS-AEMA: Chondroitin sulfate amino ethyl methacrylate; DOX: Adriamycin; DLM: Decellularized liver matrix; DFO: Desferrioxamine; DN: Double-network; DBM: Demineralized bone matrix; DA: Dopamine; dHAP: Decellularized human amniotic particles; ECM: Extracellular matrix; EHS: Engelbreth-HolmSwarm; ECFCs: Endothelial colony-forming cells; GelMA: Gelatin methacrylated; GFP: Green fluorescent protein; GFs: Growth factors; hBMSCs: Human bone marrow stromal cells; HUVECs: Human umbilical vein endothelial cells; HASCs: Human adipose-derived stem cells; HNTs: Halloysite nanotubes; HPDLSCs: Human periodontal ligament stem cells; hDPSCs: Human dental pulp stem cells; HiPSCs: Human induced pluripotent stem cells; hCPCs: Human cardiac progenitor cells; HAMA: Methacrylated hyaluronic acid; HEM: Human melanocytes; HaCat: Human keratinocytes; HDF: Human dermal fifibroblasts; hBM-MPCs: Human bone marrow-derived mesenchymal progenitor cells; HCASMCs: Human coronary artery smooth muscle cells; HFF: Human fibroblasts; HANW: Hydroxyapatite nanowires; HKs: Human corneal keratocytes; L929: Mouse osteoblast cell line; Lip: Liposome; LSSCs: Limbal stromal stem cells; MMP: Matrix metalloproteinase; MA: Methacrylamide; MNPs: Magnetic nanoparticles; MENPs: Magnetoelectric nanoparticles; MNs: Microneedles; MC3T3-E1: Mouse osteoblast cell line; MBGNs: Mesoporous bioactive glass nanoparticles; MeTro: Methacryloyl-substituted recombinant human tropoelastin; MPEG: Poly (ethylene glycol); MeCS: Methacrylated chondroitin sulfate; NIPAM: N-isopropylacrylamide; nHA: Nanohydroxyapatite;

NDs: Nanodiamonds; NRVMs: Neonatal rat ventricular myocytes; NSCs: Neural stem cells; OGP: Osteogenic growth peptides; OCP: Octacalcium phosphate; ODMA: Oligomers of dopamine methacrylate; PEDOT: Poly (3,4ethylenedioxythiophene); PSS: Poly (styrenesulfonate); PLGA: Poly (lactic-coglycolic acid); PEGDA: Poly (ethyleneglycol)diacrylate; PLL: Poly-L-lysine; PACG: Cleavable poly(N-acryloyl 2-glycine); PECL: Poly ( $\varepsilon$-caprolactone)-poly (ethylene glycol); Ppy/ES: Polypyrrole/electrospun; PdMGSMW: Palladiumbased metallic glass submicron wires; PAM: Polyacrylamide; PEG: Poly (ethylene glycol); RGD: Arginine-glycine-aspartic acid; rhBMP-2: Recombinant 
Human Bone Morphogenetic Protein-2; rGO: Reduced graphene oxide; SCAP: Stem cells from the apical papilla; TA: Tannic acid; UV: Ultraviolet; UCMSCs: Human umbilical cord mesenchymal stem cells; VEGF: Vascular endothelial growth factor; 26SCS: 2-N, 6-O-sulfated chitosan

\section{Acknowledgements}

This work was supported by the Natural Science Foundation of China (81930051), Shanghai Municipal Education Commission-Gaofeng Clinical Medicine Grant Support (20171906) and Shanghai talent development fund (2018099).

\section{Authors' contributions}

Wenguo cui made substantial contributions to conception and design of this review and critically revised the article for important intellectual content. Lei Xiang collected and summarized references and wrote this manuscript. All authors read and approved the final manuscript.

\section{Funding}

Natural Science Foundation of China (81930051).

Shanghai Municipal Education Commission-Gaofeng Clinical Medicine Grant Support (20171906).

Shanghai talent development fund (2018099).

\section{Availability of data and materials}

Not applicable.

\section{Competing interests}

The authors declare that they have no competing interests.

Received: 20 May 2020 Accepted: 2 October 2020

\section{Published online: 15 February 2021}

\section{References}

1. Young S, Wong M, Tabata Y, Mikos AG. Gelatin as a delivery vehicle for the controlled release of bioactive molecules. J Control Release. 2005;109(1-3): 256-74.

2. Maurer PH II. Antigenicity of gelatin in rabbits and other species. J Exp Med. 1954;100(5):515-23.

3. Miri AK, Hosseinabadi HG, Cecen B, Hassan S, Zhang YS. Permeability mapping of gelatin methacryloyl hydrogels. Acta Biomater. 2018;77:38-47.

4. Van Den Bulcke Al, Bogdanov B, De Rooze N, Schacht EH, Cornelissen M Berghmans H. Structural and rheological properties of methacrylamide modified gelatin hydrogels. Biomacromolecules. 2000;1(1):31-8.

5. Ludwig PE, Huff TJ, Zuniga JM. The potential role of bioengineering and three-dimensional printing in curing global corneal blindness. J Tissue Eng. 2018;9:2041731418769863.

6. Lu S, Cuzzucoli F, Jiang J, et al. Development of a biomimetic liver tumoron-a-chip model based on decellularized liver matrix for toxicity testing. Lab Chip. 2018;18(22):3379-92.

7. Nichol JW, Koshy ST, Bae H, Hwang CM, Yamanlar S, Khademhosseini A. Cell-laden microengineered gelatin methacrylate hydrogels. Biomaterials. 2010;31(21):5536-44.

8. Liu Y, Chan-Park MB. A biomimetic hydrogel based on methacrylated dextran-graft-lysine and gelatin for 3D smooth muscle cell culture. Biomaterials. 2010;31(6):1158-70.

9. Benton JA, DeForest CA, Vivekanandan V, Anseth KS. Photocrosslinking of gelatin macromers to synthesize porous hydrogels that promote valvular interstitial cell function. Tissue Eng Part A. 2009;15(11):3221-30.

10. Celikkin N, Mastrogiacomo S, Jaroszewicz J, Walboomers XF, Swieszkowski W. Gelatin methacrylate scaffold for bone tissue engineering: the influence of polymer concentration. J Biomed Mater Res A. 2018;106(1):201-9.

11. Monteiro N, Thrivikraman G, Athirasala A, et al. Photopolymerization of cellladen gelatin methacryloyl hydrogels using a dental curing light for regenerative dentistry. Dent Mater. 2018;34(3):389-99.

12. Coutinho DF, Sant SV, Shin H, et al. Modified gellan gum hydrogels with tunable physical and mechanical properties. Biomaterials. 2010;31(29):7494502.

13. Jensen SS, Terheyden $\mathrm{H}$. Bone augmentation procedures in localized defects in the alveolar ridge: clinical results with different bone grafts and bone-substitute materials. Int J Oral Maxillofac Implants. 2009;24(Suppl):21836.
14. Griffith $L G$, Naughton G. Tissue engineering--current challenges and expanding opportunities. Science. 2002;295(5557):1009-14.

15. Atala A. Engineering organs. Curr Opin Biotechnol. 2009;20(5):575-92.

16. Dong Z, Yuan Q, Huang K, Xu W, Liu G, Gu Z. Gelatin methacryloyl (GelMA)based biomaterials for bone regeneration. RSC Adv. 2019;9(31):17737-44.

17. Lee BH, Lum N, Seow LY, Lim PQ, Tan LP. Synthesis and Characterization of Types A and B Gelatin Methacryloyl for Bioink Applications. Materials (Basel). 2016;9(10):797.

18. Shirahama H, Lee BH, Tan LP, Cho NJ. Precise tuning of facile one-pot gelatin methacryloyl (GelMA) synthesis. Sci Rep. 2016;6:31036.

19. Hoch E, Schuh C, Hirth T, Tovar GE, Borchers K. Stiff gelatin hydrogels can be photo-chemically synthesized from low viscous gelatin solutions using molecularly functionalized gelatin with a high degree of methacrylation. $J$ Mater Sci Mater Med. 2012;23(11):2607-17.

20. Zhao X, Lang $Q$, Yildirimer $L$, et al. Photocrosslinkable gelatin hydrogel for epidermal tissue engineering. Adv Healthc Mater. 2016;5(1):108-18.

21. Chen YC, Lin RZ, Qi H, et al. Functional human vascular network generated in photocrosslinkable gelatin methacrylate hydrogels. Adv Funct Mater. 2012;22(10):2027-39..

22. Schuurman W, Levett PA, Pot MW, et al. Gelatin-methacrylamide hydrogels as potential biomaterials for fabrication of tissue-engineered cartilage constructs. Macromol Biosci. 2013;13(5):551-61.

23. Colosi C, Shin SR, Manoharan V, et al. Microfluidic bioprinting of heterogeneous 3D tissue constructs using low-viscosity bioink. Adv Mater. 2016;28(4):677-84

24. Vlierberghe SV, Cnudde V, Dubruel P, et al. Porous gelatin hydrogels: 1. Cryogenic formation and structure analysis. Biomacromolecules. 2007;8(2):331-7.

25. Lee Y, Lee JM, Bae PK, Chung IY, Chung BH, Chung BG. Photo-crosslinkable hydrogel-based 3D microfluidic culture device. Electrophoresis. 2015;36(7-8): 994-1001.

26. Van Vlierberghe S, Dubruel P, Schacht E. Effect of cryogenic treatment on the rheological properties of gelatin hydrogels. J Bioact Compat Polym. 2010;25(5):498-512.

27. Lin R-Z, Chen Y-C, Moreno-Luna R, Khademhosseini A, Melero-Martin JM. Transdermal regulation of vascular network bioengineering using a photopolymerizable methacrylated gelatin hydrogel. Biomaterials. 2013; 34(28):6785-96.

28. Kong B, Chen Y, Liu R, et al. Fiber reinforced GelMA hydrogel to induce the regeneration of corneal stroma. Nat Commun. 2020;11(1):1435.

29. Golafshan N, Rezahasani R, Tarkesh Esfahani M, Kharaziha M, Khorasani SN Nanohybrid hydrogels of laponite: PVA-alginate as a potential wound healing material. Carbohydr Polym. 2017;176:392-401.

30. Oh J, Kim B. Mucoadhesive and pH-responsive behavior of gelatin containing hydrogels for protein drug delivery applications. Korea-Aust Rheol J. 2020;32(1):41-6.

31. Tan H, Chu CR, Payne KA, Marra KG. Injectable in situ forming biodegradable chitosan-hyaluronic acid based hydrogels for cartilage tissue engineering. Biomaterials. 2009;30(13):2499-506.

32. Krishnamoorthy S, Wadnap S, Noorani B, Xu H, Xu C. Investigation of gelatin methacrylate working curves in dynamic optical projection stereolithography of vascular-like constructs. Eur Polym J. 2020;124:109487.

33. Xiao $Y$, Reis $L A$, Feric $N$, et al. Diabetic wound regeneration using peptidemodified hydrogels to target re-epithelialization. Proc Natl Acad Sci U S A. 2016;113(40):E5792-801

34. Wang $\mathrm{T}$, Wang J, He X, et al. An ambient curable coating material based on the michael addition reaction of acetoacetylated castor oil and multifunctional acrylate. Coatings. 2019;9:37.

35. Nicolas F. Denatured thiolated collagen. II.Cross-linking by oxidation. Biomaterials. 1997;18(11):815-21.

36. Ning Z, Tan B, Chen B, et al. Precisely controlled delivery of abaloparatide through injectable hydrogel to promote bone regeneration. Macromol Biosci. 2019;19(6):e1900020.

37. Samorezov JE, Headley EB, Everett CR, Alsberg E. Sustained presentation of BMP-2 enhances osteogenic differentiation of human adipose-derived stem cells in gelatin hydrogels. J Biomed Mater Res A. 2016;104(6):1387-97.

38. Vigata M, Meinert C, Pahoff S, Bock N, Hutmacher DW. Gelatin methacryloyl hydrogels control the localized delivery of albumin-bound paclitaxel. Polymers (Basel). 2020;12(2):501.

39. Cheng R, Yan Y, Liu H, et al. Mechanically enhanced lipo-hydrogel with controlled release of multi-type drugs for bone regeneration. Appl Mater Today. 2018;12:294-308. 
40. Samanipour R, Wang T, Werb M, et al. Ferritin nanocage conjugated hybrid hydrogel for tissue engineering and drug delivery applications. ACS Biomater-sci Eng. 2020;6(1):277-87.

41. Kang MG, Lee MY, Cha JM, et al. Nanogels derived from fish gelatin: application to drug delivery system. Mar Drugs. 2019;17(4):246.

42. Modaresifar K, Hadjizadeh A, Niknejad H. Design and fabrication of GeIMA/ chitosan nanoparticles composite hydrogel for angiogenic growth factor delivery. Artif Cells Nanomed Biotechnol. 2018;46(8):1799-808.

43. Oktay S, Alemdar N. Electrically controlled release of 5-fluorouracil from conductive gelatin methacryloyl-based hydrogels. J Appl Polym Sci. 2019;136(1):46914.

44. Li Y, Yan D, Fu F, et al. Composite core-shell microparticles from microfluidics for synergistic drug delivery. SciChina Mater. 2017;60(6):543-53.

45. Shrimali $P$, Peter $M$, Singh A, et al. Efficient in situ gene delivery via PEG diacrylate matrices. Biomater Sci. 2018;6(12):3241-50.

46. Lai TC, Yu J, Tsai WB. Gelatin methacrylate/carboxybetaine methacrylate hydrogels with tunable crosslinking for controlled drug release. J Mater Chem B. 2016:4(13):2304-13.

47. Sun $X$, Zhao $X$, Zhao L, et al. Development of a hybrid gelatin hydrogel platform for tissue engineering and protein delivery applications. J Mater Chem B. 2015;3(30):6368-76.

48. Jalili NA, Jaiswal MK, Peak CW, Cross LM, Gaharwar AK. Injectable nanoengineered stimuli-responsive hydrogels for on-demand and localized therapeutic delivery. Nanoscale. 2017;9(40):15379-89.

49. Dong M, Wang X, Chen X-Z, et al. 3D-printed soft magnetoelectric microswimmers for delivery and differentiation of neuron-like cells. Adv Funct Mater. 2020;30(17):1910323.

50. Luo Z, Sun W, Fang J, et al. Biodegradable gelatin methacryloyl microneedles for transdermal drug delivery. Adv Healthc Mater. 2019;8(3): e1801054.

51. Choi YH, Kim SH, Kim IS, Kim K, Kwon SK, Hwang NS. Gelatin-based microhydrogel carrying genetically engineered human endothelial cells for neovascularization. Acta Biomater. 2019:95:285-96.

52. Monteiro N, He W, Franca CM, Athirasala A, Bertassoni LE. Engineering microvascular networks in led light-cured cell-laden hydrogels. ACS Biomater-Sci Eng. 2018;4(7):2563-70.

53. Zhao Q, Wang J, Cui H, Chen H, Wang Y, Du X. Programmed shapemorphing scaffolds enabling facile 3d endothelialization. Adv Funct Mater. 2018;28(29):1801027.

54. Zhang YS, Pi Q, van Genderen AM. Microfluidic bioprinting for engineering vascularized tissues and organoids. J Vis Exp. 2017:126:e55957.

55. Kim P, Yuan A, Nam KH, Jiao A, Kim DH. Fabrication of poly (ethylene glycol): gelatin methacrylate composite nanostructures with tunable stiffness and degradation for vascular tissue engineering. Biofabrication. 2014;6(2):024112.

56. Liew WEN, Zhang Y. Cell-laden gelatin methacryloyl fibres fabricated using bessel beams for controlled endothelial cord formation. Biomed Phys Eng Express. 2018:4:045009.

57. Cui $H$, Zhu W, Huang $Y$, et al. In vitro and in vivo evaluation of $3 D$ bioprinted small-diameter vasculature with smooth muscle and endothelium. Biofabrication. 2019;12(1):015004.

58. Yang L, Shridhar SV, Gerwitz M, Soman P. An in vitro vascular chip using 3D printing-enabled hydrogel casting. Biofabrication. 2016;8(3):035015.

59. Tao J, Zhang J, Du T, et al. Rapid 3D printing of functional nanoparticleenhanced conduits for effective nerve repair. Acta Biomater. 2019;90:49-59.

60. Zhou X, Cui H, Nowicki M, et al. Three-dimensional-bioprinted dopaminebased matrix for promoting neural regeneration. ACS Appl Mater Interfaces. 2018;10(10):8993-9001.

61. Fan L, Liu C, Chen X, et al. Directing induced pluripotent stem cell derived neural stem cell fate with a three-dimensional biomimetic hydrogel for spinal cord injury repair. ACS Appl Mater Interfaces. 2018;10(21):17742-55.

62. Chen C, Tang J, Gu Y, et al. Bioinspired hydrogel electrospun fibers for spinal cord regeneration. Adv Funct Mater. 2019;29(4):1806899.

63. Wu Y, Xiang Y, Fang J, et al. The influence of the stiffness of GelMA substrate on the outgrowth of PC12 cells. Biosci Rep. 2019;39(1): BSR20181748.

64. Gong H, Fei H, Xu Q, Gou M, Chen HH. 3D-engineered GelMA conduit filled with ECM promotes regeneration of peripheral nerve. J Biomed Mater Res A. 2020;108(3):805-13.

65. Soucy JR, Shirzaei Sani E, Portillo Lara R, et al. Photocrosslinkable gelatin/ tropoelastin hydrogel adhesives for peripheral nerve repair. Tissue Eng Part A. 2018;24(17-18):1393-405.
66. Mukhatyar V, Karumbaiah L, Yeh J, Bellamkonda R. Tissue engineering strategies designed to realize the endogenous regenerative potential of peripheral nerves. Adv Mater. 2009;21(46):4670-9.

67. Griffin JW, Hogan MV, Chhabra AB, Deal DN. Peripheral nerve repair and reconstruction. J Bone Joint Surg Am. 2013;95(23):2144-51.

68. Wang Y, Ma M, Zhang L, Gao Y, Zhang B, Guo Y. Fabrication of bi-layer photocrosslinked GeIMA/PEGDA fibrous membrane for guided bone regeneration materials. Mater Lett. 2019;249:112-5.

69. Tang W, Yu Y, Wang J, et al. Enhancement and orchestration of osteogenesis and angiogenesis by a dual-modular design of growth factors delivery scaffolds and 26SCS decoration. Biomaterials. 2020;232:119645.

70. Chen H, Yan Y, Qi J, Deng L, Cui W. Sustained delivery of desferrioxamine via liposome carriers in hydrogel for combining angiogenesis and osteogenesis in bone defects reconstruction. J Control Release. 2017;259: e79.

71. Liu J, Li L, Suo H, Yan M, Yin J, Fu J. 3D printing of biomimetic multi-layered GeIMA/nHA scaffold for osteochondral defect repair. Mater Design. 2019; 171:107708.

72. Zheng J, Zhao F, Zhang W, et al. Sequentially-crosslinked biomimetic bioactive glass/gelatin methacryloyl composites hydrogels for bone regeneration. Mater Sci Eng C Mater Biol Appl. 2018;89:119-27.

73. Lee D, Choi EJ, Lee SE, et al. Injectable biodegradable gelatin-methacrylate/ $\beta$-tricalcium phosphate composite for the repair of bone defects. Chem Eng J. 2019;365:30-9.

74. Ou Q, Huang K, Fu C, et al. Nanosilver-incorporated halloysite nanotubes/ gelatin methacrylate hybrid hydrogel with osteoimmunomodulatory and antibacterial activity for bone regeneration. Chem Eng J. 2020;382:123019.

75. Xin T, Gu Y, Cheng R, et al. Inorganic strengthened hydrogel membrane as regenerative periosteum. ACS Appl Mater Interfaces. 2017;9(47):41168-80.

76. Xin T, Mao J, Liu L, et al. Programmed sustained release of recombinant human bone morphogenetic protein-2 and inorganic ion composite hydrogel as artificial periosteum. ACS Appl Mater Interfaces. 2020;12(6): 6840-51.

77. Ma Y, Ji Y, Zhong T, et al. Bioprinting-based pdlsc-ecm screening for in vivo repair of alveolar bone defect using cell-laden, injectable and photocrosslinkable hydrogels. ACS Biomater Sci Eng. 2017:3(12):3534-45.

78. Huang $\mathrm{K}, \mathrm{Ou} \mathrm{Q}$, Xie Y, et al. Halloysite nanotube based scaffold for enhanced bone regeneration. ACS Biomater-Sci Eng. 2019:5(8):4037-47.

79. Qiao Y, Liu X, Zhou X, et al. Gelatin templated polypeptide co-cross-linked hydrogel for bone regeneration. Adv Healthc Mater. 2020;9(1):e1901239.

80. Gao F, Xu Z, Liang Q, et al. Osteochondral regeneration with 3d-printed biodegradable high-strength supramolecular polymer reinforced-gelatin hydrogel scaffolds. Adv Sci. 2019;6(15):1900867.

81. Liu L, Li X, Shi X, Wang Y. Injectable alendronate-functionalized GelMA hydrogels for mineralization and osteogenesis. RSC Adv. 2018;8(40):22764-76.

82. Zhang $Y$, Chen M, Tian J, et al. In situ bone regeneration enabled by a biodegradable hybrid double-network hydrogel. Biomater Sci. 2019;7(8):3266-76.

83. Gu L, Zhang J, Li L, Du Z, Cai Q, Yang X. Hydroxyapatite nanowire composited gelatin cryogel with improved mechanical properties and cell migration for bone regeneration. Biomed Mater. 2019;14(4):045001.

84. Anada T, Pan CC, Stahl AM, et al. Vascularized bone-mimetic hydrogel constructs by $3 \mathrm{~d}$ bioprinting to promote osteogenesis and angiogenesis. Int J Mol Sci. 2019:20(5):1096.

85. Thakur T, Xavier JR, Cross L, et al. Photocrosslinkable and elastomeric hydrogels for bone regeneration. J Biomed Mater Res A. 2016;104(4):879-88.

86. Wu W, Dai Y, Liu H, et al. Local release of gemcitabine via in situ UVcrosslinked lipid-strengthened hydrogel for inhibiting osteosarcoma. Drug Deliv. 2018;25(1):1642-51.

87. Wang Y, Ma M, Wang J, et al. Development of a photo-crosslinking, biodegradable GeIMA/PEGDA hydrogel for guided bone regeneration materials. Materials (Basel). 2018;11(8):1345.

88. Kwon S, Lee SS, Sivashanmugam A, et al. Bioglass-incorporated methacrylated gelatin cryogel for regeneration of bone defects. Polymers (Basel). 2018;10(8):914.

89. Celikkin N, Mastrogiacomo S, Walboomers XF, Swieszkowski W. Enhancing $\mathrm{x}$-ray attenuation of $3 \mathrm{~d}$ printed gelatin methacrylate (gelma) hydrogels utilizing gold nanoparticles for bone tissue engineering applications. Polymers (Basel). 2019;11(2):367.

90. Byambaa B, Annabi N, Yue K, et al. Bioprinted osteogenic and vasculogenic patterns for engineering 3D bone tissue. Adv Healthc Mater. 2017;6(16). https://doi.org/10.1002/adhm.201700015. 
91. Pacelli S, Maloney R, Chakravarti AR, et al. Controlling adult stem cell behavior using nanodiamond-reinforced hydrogel: implication in bone regeneration therapy. Sci Rep. 2017;7(1):6577.

92. Moghanian A, Portillo-Lara R, Shirzaei Sani E, Konisky H, Bassir SH, Annabi N. Synthesis and characterization of osteoinductive visible light-activated adhesive composites with antimicrobial properties. J Tissue Eng Regen Med. 2020;14(1):66-81.

93. Ramis JM, Blasco-Ferrer M, Calvo J, et al. Improved physical and osteoinductive properties of demineralized bone matrix by gelatin methacryloyl formulation. J Tissue Eng Regen Med. 2020;14(3):475-85.

94. Zhao X, Liu S, et al. Injectable stem cell-laden Photocrosslinkable microspheres fabricated using microfluidics for rapid generation of Osteogenic tissue constructs. Adv Funct Mater. 2016;26:2809-19.

95. Han M-E, Kim S-H, Kim HD, et al. Extracellular matrix-based cryogels for cartilage tissue engineering. Int J Biol Macromol. 2016:93:1410-9.

96. Mouser VHM, Dautzenberg NMM, Levato R, et al. Ex vivo model unravelling cell distribution effect in hydrogels for cartilage repair. Altex. 2018;35(1):65-76.

97. Mouser VHM, Levato R, Mensinga A, Dhert WJA, Gawlitta D, Malda J. Bio-ink development for three-dimensional bioprinting of hetero-cellular cartilage constructs. Connect Tissue Res. 2020;61(2):137-51.

98. $\mathrm{Lu} \mathrm{H}, \mathrm{Xu}$ J, Xiong $\mathrm{L}$, et al. Biohybrid methacrylated gelatin/polyacrylamide hydrogels for cartilage repair. J Mater Chem B. 2017;5:731-41.

99. Gan D, Xu T, Xing W, et al. Mussel-inspired dopamine oligomer intercalated tough and resilient gelatin methacryloyl (GelMA) hydrogels for cartilage regeneration. J Mater Chem B. 2019;7(10):1716-25.

100. Pahoff S, Meinert C, Bas O, Nguyen L, Klein TJ, Hutmacher DW. Effect of gelatin source and photoinitiator type on chondrocyte redifferentiation in gelatin methacryloyl-based tissue-engineered cartilage constructs. J Mater Chem B. 2019;7(10):1761-72.

101. Chung JHY, Kade JC, Jeiranikhameneh A, et al. 3D hybrid printing platform for auricular cartilage reconstruction. Biomed Phys Eng Express. 2020;6(3): 035003

102. Costantini M, Idaszek J, Szöke K, et al. 3D bioprinting of BM-MSCs-loaded ECM biomimetic hydrogels for in vitro neocartilage formation. Biofabrication. 2016:8(3):035002.

103. Suo H, Li L, Zhang C, et al. Glucosamine-grafted methacrylated gelatin hydrogels as potential biomaterials for cartilage repair. J Biomed Mater Res B. 2020;108(3):990-9.

104. Pirosa A, Clark KL, Tan J, et al. Modeling appendicular skeletal cartilage development with modified high-density micromass cultures of adult human bone marrow-derived mesenchymal progenitor cells. Stem Cell Res Ther. 2019;10(1):388

105. Zhu W, Cui H, Boualam B, et al. 3D bioprinting mesenchymal stem cellladen construct with core-shell nanospheres for cartilage tissue engineering. Nanotechnology. 2018;29(18):185101.

106. Ahadian S, Banan Sadeghian R, Yaginuma S, et al. Hydrogels containing metallic glass sub-micron wires for regulating skeletal muscle cell behaviour. Biomater Sci. 2015;3(11):1449-58.

107. García-Lizarribar A, Fernández-Garibay X, Velasco-Mallorquí F, Castaño AG, Samitier J, Ramon-Azcon J. Composite biomaterials as long-lasting scaffolds for 3D bioprinting of highly aligned muscle tissue. Macromol Biosci. 2018; 18(10):e1800167

108. Seyedmahmoud R, Celebi-Saltik B, Barros N, et al. Three-dimensiona bioprinting of functional skeletal muscle tissue using gelatinmethacryloylalginate bioinks. Micromachines (Basel). 2019:10(10):679.

109. Ahadian S, Ramón-Azcón J, Estili M, et al. Hybrid hydrogels containing vertically aligned carbon nanotubes with anisotropic electrical conductivity for muscle myofiber fabrication. Sci Rep UK. 2014;4(1):4271.

110. Ostrovidov S, Ahadian S, Ramon-Azcon J, et al. Three-dimensional co-culture of $\mathrm{C} 2 \mathrm{C} 12 / \mathrm{PC} 12$ cells improves skeletal muscle tissue formation and function J Tissue Eng Regen Med. 2017;11(2):582-95.

111. Ebrahimi M, Ostrovidov S, Salehi S, Kim SB, Bae H, Khademhosseini A. Enhanced skeletal muscle formation on microfluidic spun gelatin methacryloyl (GelMA) fibres using surface patterning and agrin treatment. J Tissue Eng and Regen M. 2018;12(11):2151-63.

112. Ostrovidov S, Hosseini V, Ahadian S, et al. Skeletal muscle tissue engineering: methods to form skeletal myotubes and their applications. Tissue Eng Part B Rev. 2014;20(5):403-36.

113. MacDonald RA, Voge CM, Kariolis M, Stegemann JP. Carbon nanotubes increase the electrical conductivity of fibroblast-seeded collagen hydrogels. Acta Biomater. 2008;4(6):1583-92.
114. Sun H, Tang J, Mou Y, et al. Carbon nanotube-composite hydrogels promote intercalated disc assembly in engineered cardiac tissues through B1-integrin mediated FAK and RhoA pathway. Acta Biomater. 2017;48:88-99.

115. Chakravarti AR, Pacelli $S$, Alam $P$, et al. Pre-conditioning stem cells in a biomimetic environment for enhanced cardiac tissue repair: in vitro and in vivo analysis. Cell Mol Bioeng. 2018;11(5):321-36.

116. Alemdar N, Leijten J, Camci-Unal G, et al. Oxygen-generating photo-crosslinkable hydrogels support cardiac progenitor cell survival by reducing hypoxia-induced necrosis. ACS Biomater- Sci Eng. 2017;3(9):1964-71.

117. Kerscher P, Kaczmarek JA, Head SE, et al. Direct production of human cardiac tissues by pluripotent stem cell encapsulation in gelatin methacryloyl. ACS Biomater Sci Eng. 2017;3(8):1499-509.

118. Li L, Chen Z, Shao C, Sun L, Sun L, Zhao Y. Graphene hybrid anisotropic structural color film for cardiomyocytes' monitoring. Adv Funct Mater. 2020; 30(3):1906353.

119. Bejleri D, Streeter BW, Nachlas ALY, et al. A bioprinted cardiac patch composed of cardiac-specific extracellular matrix and progenitor cells for heart repair. Adv Healthc Mater. 2018;7(23):1800672.

120. Walker BW, Lara RP, Yu CH, et al. Engineering a naturally-derived adhesive and conductive cardiopatch. Biomaterials. 2019;207:89-101.

121. He Y, Ye G, Song C, et al. Mussel-inspired conductive nanofibrous membranes repair myocardial infarction by enhancing cardiac function and revascularization. Theranostics. 2018;8(18):5159-77.

122. Chan V, Raman R, Cvetkovic C, Bashir R. Enabling microscale and nanoscale approaches for bioengineered cardiac tissue. ACS Nano. 2013;7(3):1830-7.

123. Bakhsheshi-Rad HR, Ismail AF, Aziz M, et al. Antibacterial activity and in vivo wound healing evaluation of polycaprolactone-gelatin methacryloylcephalexin electrospun nanofibrous. Mater Lett. 2019;256:126618.

124. Eke G, Mangir N, Hasirci N, MacNeil S, Hasirci V. Development of a UV crosslinked biodegradable hydrogel containing adipose derived stem cells to promote vascularization for skin wounds and tissue engineering. Biomaterials. 2017:129:188-98.

125. Liu B, Wang Y, Miao Y, et al. Hydrogen bonds autonomously powered gelatin methacrylate hydrogels with super-elasticity, self-heal and underwater self-adhesion for sutureless skin and stomach surgery and Eskin. Biomaterials. 2018:171:83-96.

126. Zhang X, Li J, Ye P, Gao G, Hubbell K, Cui X. Coculture of mesenchymal stem cells and endothelial cells enhances host tissue integration and epidermis maturation through AKT activation in gelatin methacryloyl hydrogel-based skin model. Acta Biomater. 2017;59:317-26.

127. Chen H, Guo L, Wicks J, et al. Quickly promoting angiogenesis by using a DFO-loaded photo-crosslinked gelatin hydrogel for diabetic skin regeneration. J Mater Chem B. 2016:4(21):3770-81.

128. Annabi N, Rana D, Shirzaei Sani E, et al. Engineering a sprayable and elastic hydrogel adhesive with antimicrobial properties for wound healing. Biomaterials. 2017;139:229-43.

129. Zhao $X$, Sun $X$, Yildirimer $L$, et al. Cell infiltrative hydrogel fibrous scaffolds for accelerated wound healing. Acta Biomater. 2017;49:66-77.

130. Uehara M, Li X, Sheikhi A, et al. Anti-IL-6 eluting immunomodulatory biomaterials prolong skin allograft survival. Sci Rep. 2019;9(1):6535.

131. Shi $Y$, Xing TL, Zhang HB, et al. Tyrosinase-doped bioink for 3D bioprinting of living skin constructs. Biomed Mater. 2018;13(3):035008.

132. Sun $X$, Lang $Q$, Zhang $H$, Cheng $L$, et al. Electrospun Photocrosslinkable hydrogel fibrous scaffolds for rapid in vivo vascularized skin flap regeneration. Adv Funct Mater. 2017;27:1604617.

133. Uyanıklar M, Günal G, Tevlek A, Hosseinian P, Aydin HM. Hybrid cornea: cell laden hydrogel incorporated decellularized matrix. ACS Biomater-Sci Eng. 2020;6(1):122-33.

134. Kilic Bektas C, Hasirci V. Cell loaded 3D bioprinted GelMA hydrogels for corneal stroma engineering. Biomater Sci-uk. 2020;8(1):438-49.

135. Kilic Bektas C, Hasirci V. Mimicking corneal stroma using keratocyte-loaded photopolymerizable methacrylated gelatin hydrogels. J Tissue Eng Regen M 2018;12(4):e1899-910.

136. Cui J, Wang H, Shi Q, Sun T, Huang Q, Fukuda T. Multicellular co-culture in three-dimensional gelatin methacryloyl hydrogels for liver tissue engineering. Molecules. 2019;24(9):1762.

137. Zhang Q, Qian C, Xiao W, et al. Development of a visible light, cross-linked GelMA hydrogel containing decellularized human amniotic particles as a soft tissue replacement for oral mucosa repair. RSC Adv. 2019;9(32):18344-52.

138. Whitcher JP, Srinivasan M, Upadhyay MP. Corneal blindness: a global perspective. Bull World Health Organ. 2001;79(3):214-21. 
139. Kilic Bektas C, Burcu A, Gedikoglu G, Telek HH, Ornek F, Hasirci V. Methacrylated gelatin hydrogels as corneal stroma substitutes: in vivo study. J Biomater Sci Polym Ed. 2019;30(18):1803-21.

140. Zhang $L$, Xiang $Y$, Zhang $H$, et al. A biomimetic $3 d$-self-forming approach for microvascular scaffolds. Adv Sci. 2020;7(9):1903553.

\section{Publisher's Note}

Springer Nature remains neutral with regard to jurisdictional claims in published maps and institutional affiliations.

Submit your manuscript to a SpringerOpen ${ }^{\odot}$ journal and benefit from:

- Convenient online submission

- Rigorous peer review

- Open access: articles freely available online

High visibility within the field

- Retaining the copyright to your article

Submit your next manuscript at $\boldsymbol{\wedge}$ springeropen.com 